\section{A) Check for updates}

Cite this: Polym. Chem., 2021, 12 3661

\title{
New horizons for carbon dots: quantum nano-photoinitiating catalysts for cationic photopolymerization and three-dimensional (3D) printing under visible light $\uparrow$
}

\author{
Wiktoria Tomal, (D) a Tomasz Świergosz, (D) ${ }^{b}$ Maciej Pilch, (D) ${ }^{a}$ Wiktor Kasprzyk (D) ${ }^{a}$ \\ and Joanna Ortyl (iD *a,c
}

\begin{abstract}
Herein, the application of various citric acid-based carbon dots (CDs) as efficient photosensitizers of iodonium salts in initiating the light-induced cationic polymerization process was investigated. Hence, carbon dots prepared from citric acid only (CA-CDs) and doped with amino ( $\mathrm{N}$-doped-CA-CDs) and sulphur (N,S-doped-CA-CDs) precursors have been utilized as universal iodonium salt photosensitizers for the cationic photopolymerization of vinyl and epoxy monomers and the free-radical photopolymerization of acrylates including the formation of hydrogels. Furthermore, carbon dots have been found to be intriguing nano-photoinitiating catalysts that exhibit extraordinary properties and can be successfully applied in 3D printing under visible light.
\end{abstract}

Received 19th February 2021, Accepted 7th May 2021

DOI: $10.1039 / \mathrm{d} 1$ py00228g rsc.li/polymers are currently working on new molecules, the so-called iodine salt photosensitizers, to efficiently transfer electrons to the iodine salt and thus effectively trigger light-initiated polymerization. ${ }^{19}$ Many types of compounds are available as effective photosensitizers of iodine salts, including biphenyl, ${ }^{20,21}$ terphenyl, ${ }^{1,22,28}$ thioxanthone, ${ }^{23}$ pyridine $^{24-26}$ or 1,8-naphthalimide $^{27}$ derivatives. However, it is a constant challenge to develop more "greener" sensitizers derived from natural resources that are non-toxic and highly effective and can be successfully exploited in biomedical applications.

Carbon dots are one group among innovative materials that are attracting more and more scientific attention. ${ }^{28,29}$ These are materials that exhibit unique optical, ${ }^{30}$ photoluminescence $^{31,32}$ and chemiluminescence properties. ${ }^{29,33,34}$ Due to their numerous advantages, including exceptional water solubility, ${ }^{35}$ biocompatibility, ${ }^{36}$ excellent chemical passivity and high photobleaching resistance, ${ }^{37}$ these materials are employed, among others, as chemical or biological sensors ${ }^{38}$ in bio-imaging ${ }^{39}$ and in biomedicine. ${ }^{40}$ The latest reports also show the elaboration of the fluorophore structures formed during the synthesis of carbon dots and their role in the fluorescence phenomena. ${ }^{41-45}$ Furthermore, as a result of CDs' intriguing electrochemical properties, they have been applied in photocatalysis and photopolymerization processes. ${ }^{46}$ Carbon dots doped with heteroatoms have been described for the first time by Matyjaszewski et al. as photocatalysts for reversible addition-fragmentation chain transfer (RAFT) polymerization. ${ }^{47}$ Meanwhile, Strehmel et al. proposed the utilization of carbon dots in a two-component initiation system

\footnotetext{
${ }^{a}$ Department of Biotechnology and Physical Chemistry, Faculty of Chemical Engineering and Technology, Cracow University of Technology, Warszawska 24, 31-155 Kraków, Poland. E-mail: jortyl@pk.edu.pl

${ }^{b}$ Department of Analytical Chemistry, Faculty of Chemical Engineering and Technology, Cracow University of Technology, Warszawska 24, 31-155 Kraków, Poland

${ }^{c}$ Photo HiTech Ltd, Bobrzyńskiego 14, 30-348 Cracow, Poland

$\dagger$ Electronic supplementary information (ESI) available. See DOI: 10.1039/ d1py00228g
} 
together with iodine or sulfonic salt, where $\mathrm{Cu}^{\mathrm{II}}$ was used as a ppm scale catalyst for photo-ATRP (Atom Transfer Radical Polymerization) as well as for free radical photopolymerization (FRP). ${ }^{48}$ Thus, until now, the employment of nanodots in light-initiated polymerization processes has been limited to RAFT or ATRP, ${ }^{49}$ i.e., general radical photopolymerization processes.

Herein, for the first time, we present the prospect of using citric acid-based carbon dots, doped with heteroatoms in the form of amino and sulphur compounds, as photosensitizers of iodine salts to initiate cationic polymerization processes. For this purpose, three types of carbon dots were fabricated CA-CDs, N-doped-CA-CDs, and N,S-doped-CA-CDs, and their synthesis and detailed purification procedure are reported. The required spectroscopic and electrochemical properties of these materials are presented. Moreover, carbon dots were employed in a binary initiation system for the cationic photopolymerization of vinyl monomers along with the cationic photopolymerization of ring-opening epoxy monomers. Additionally, the utilization of carbon dots as photosensitizers of iodine salt in radical photopolymerization processes involving the fabrication of hydrogel materials was investigated. Furthermore, the visible properties of C-dots were confirmed, and the possibility of their use as green photocatalysts was investigated. Ultimately, 3D printing experiments involving cationic photopolymerization with the utilization of an initiating system containing carbon dots for hydrogel materials were performed. The noted research gap in the form of the usage of carbon dots in cationic photopolymerization processes, along with the utilization of these materials for $3 \mathrm{D}$ printing, including printed hydrogels, was the motivation of this study.

\section{Experimental}

\section{Materials}

The synthesis of carbon dots was conducted using citric acid (CA) (for the fabrication of CA-CDs, CA and urea (for the fabrication of N-doped-CA-CDs), and CA and thiourea (for the fabrication of N,S-doped-CA-CDs). The following reagents were used for the carbon dot purification: $1 \mathrm{M} \mathrm{NaOH}$ and $1 \mathrm{M} \mathrm{HCl}$ aqueous solutions. All reagents were provided by Sigma Aldrich and were used as received. For photopolymerization processes, the following reagents were utilized: bis-(4-t-butylphenyl)iodonium hexafluorophosphate (Iod, Speedcure 938, Lambson, Wetherby, UK) as a commercial photoinitiator, methyl diethanolamine (MDEA, Sigma Aldrich) as a coinitiator in type-II photoinitiator systems For photopolymerization investigation, model monomers were applied: 3,4-epoxycyclohexylmethyl-3,4-epoxycyclohexane-carboxylate (CADE, Lambson, Wetherby, UK) and tri(ethylene glycol) divinyl ether (TEGDVE, Sigma Aldrich) for cationic polymerization, as well as trimethylolpropane triacrylate (TMPTA, Sigma Aldrich) for free-radical photopolymerization. Furthermore, 2-hydroxyethyl acrylate (HEA, Sigma Aldrich) was used for the preparation of photocurable hydrogels. The following additives were used to stimulate the polymerization reaction: $\mathrm{N}$-vinylcarbazole (NVK, Sigma Aldrich) and tris(trimethylsilyl) silane (TTMSS, Sigma Aldrich). The chemical structures of the utilized reagents are shown in Fig. S2. $\dagger$

\section{Carbon dot synthesis}

A one-pot bottom-up fabrication method for the synthesis of carbon dots was developed by the standard procedure for condensation reactions: ${ }^{43,44} \mathrm{CA}(0.192 \mathrm{~g}, 1 \mathrm{mmol})$ (reaction (1) in Fig. 1 and S1†); CA (0.192 g, $1 \mathrm{mmol})$ mixed with an amine precursor: urea (0.600 g, $10 \mathrm{mmol}$ ) (reaction (2) in Fig. 1 and S1 $\dagger$ ); and CA (0.192 g, $1 \mathrm{mmol})$ mixed with a sulphur containing precursor: thiourea $(0.760 \mathrm{~g}, 10 \mathrm{mmol})$ (reaction (3) in Fig. 1 and $\mathrm{S} 1 \dagger$ ) was placed in a glass vial reactor and kept at $230{ }^{\circ} \mathrm{C}$ for $2 \mathrm{~h}$ (Table S1 $\dagger$ ). Reactions were carried out on a heating block with a magnetic stirrer at $100 \mathrm{rpm}$. Afterward, the products of the synthesis were cooled down to room temperature and subjected to the purification process.

\section{Carbon dot purification procedure}

Proper purification of carbon dots was a crucial point at this stage of the investigation. Therefore, the solid particles obtained from the one-pot synthesis were treated with $1 \mathrm{M} \mathrm{NaOH}$ solution (10 $\mathrm{mL}$ of alkaline solution was used) and then subjected to ultrasonication for 30 minutes for dissolution. The obtained solutions were then neutralized with $1 \mathrm{M} \mathrm{HCl}$, until the $\mathrm{pH}$ of the tested solution dropped down to about 7 (Fig. 2).

The solution containing carbon dots, fluorophores, and other impurities was then alternately centrifuged and rinsed using ultrafiltration units (Vivaspin 20, MWCO $10 \mathrm{kDa}-$ MWCO $100 \mathrm{kDa}$ ) to remove the fluorophores and obtain a solution containing only target CDs (Fig. 3 and S3†). Additional spectroscopic studies on the quality of the carbon dot purification procedure are shown in Fig. S4. $\dagger$ The fine carbon dots

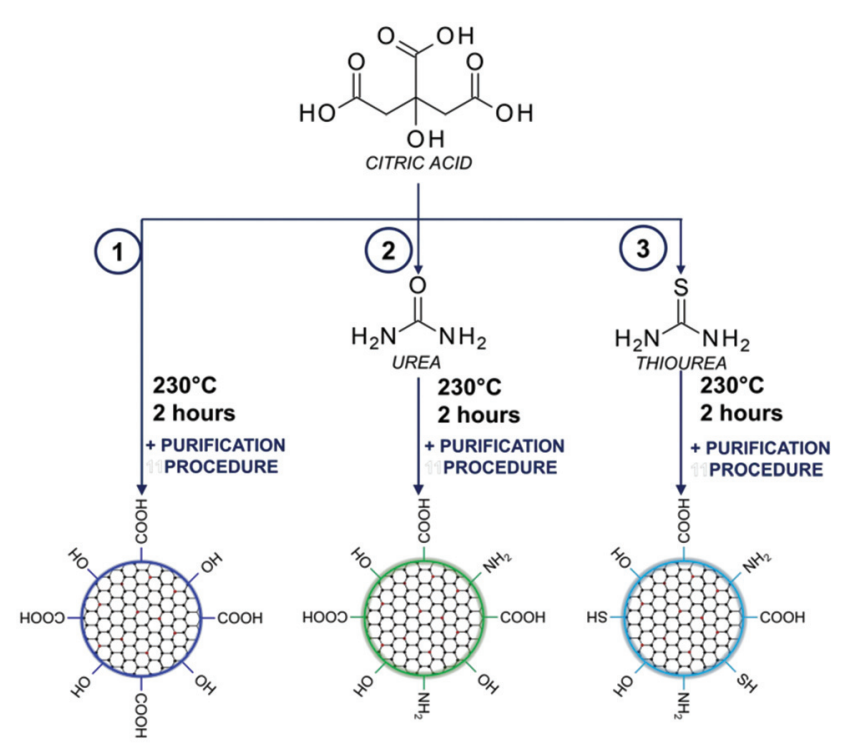

Fig. 1 Synthesis scheme of carbon dots based on the utilization of citric acid with amino and sulphuric precursors. 

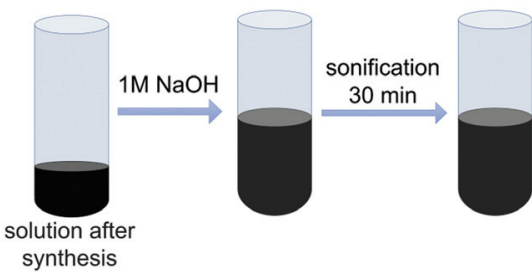

synthesis

Fig. 2 Preparation of the reaction mixture for the fluorophore carbon dot purification procedure.

were then frozen and freeze-dried to provide a dry product characterized by a wadding consistency.

\section{Determination of the size and morphology of carbon dots}

The size distribution and the average size of the prepared carbon dots were directly determined in the aqueous suspension $\left(0.1 \mathrm{mg} \mathrm{mL} \mathrm{mL}^{-1}\right)$ using dynamic light scattering (DLS) (Zetasizer Nano ZS instrument from Malvern). Due to significant aggregation of $\mathrm{CDs}$ at neutral $\mathrm{pH}$, all measurements were con- ducted at $\mathrm{pH} 10$ (NaOH was used for $\mathrm{pH}$ adjustment). A transmission electron microscope (TEM) of high resolution (Tecnai TF $20 \mathrm{X}$-TWIN $(200 \mathrm{kV})$ ) equipped with a field emission gun (FEG) was employed for the morphology analysis of the CDs. Time-resolved Tecnai TF 20 X-TWIN microscope provided $0.5 \mathrm{nA}$ intensity capability in an electron beam focused on a spot of $1 \mathrm{~nm}$ diameter (spot resolution capability of $1 \mathrm{~nm}$ diameter (spot resolution $\leq 0.22 \mathrm{~nm}$ and information limit $\leq 0.14 \mathrm{~nm}$ )).

\section{Determination of the chemical bonding and chemical composition of carbon dots}

XPS analyses were carried out in a PHI VersaProbeII Scanning XPS system using monochromatic Al $\mathrm{K}_{\alpha}(1486.6 \mathrm{eV})$ X-rays focused to a $100 \mu \mathrm{m}$ spot and scanned over the area of $400 \mu \mathrm{m} \times 400 \mu \mathrm{m}$. The photoelectron take-off angle was $45^{\circ}$ and the pass energy in the analyzer was set to $117.50 \mathrm{eV}$ for survey scans and $46.95 \mathrm{eV}$ to obtain high energy resolution spectra for the $\mathrm{C} 1 \mathrm{~s}, \mathrm{~N} 1 \mathrm{~s}, \mathrm{~S} 2 \mathrm{p}, \mathrm{Na} 1 \mathrm{~s}$ and $\mathrm{O} 1 \mathrm{~s}$ regions. A dual beam charge compensation with $7 \mathrm{eV} \mathrm{Ar}^{+}$ions and $1 \mathrm{eV}$ electrons was used to maintain a constant sample surface potential regardless

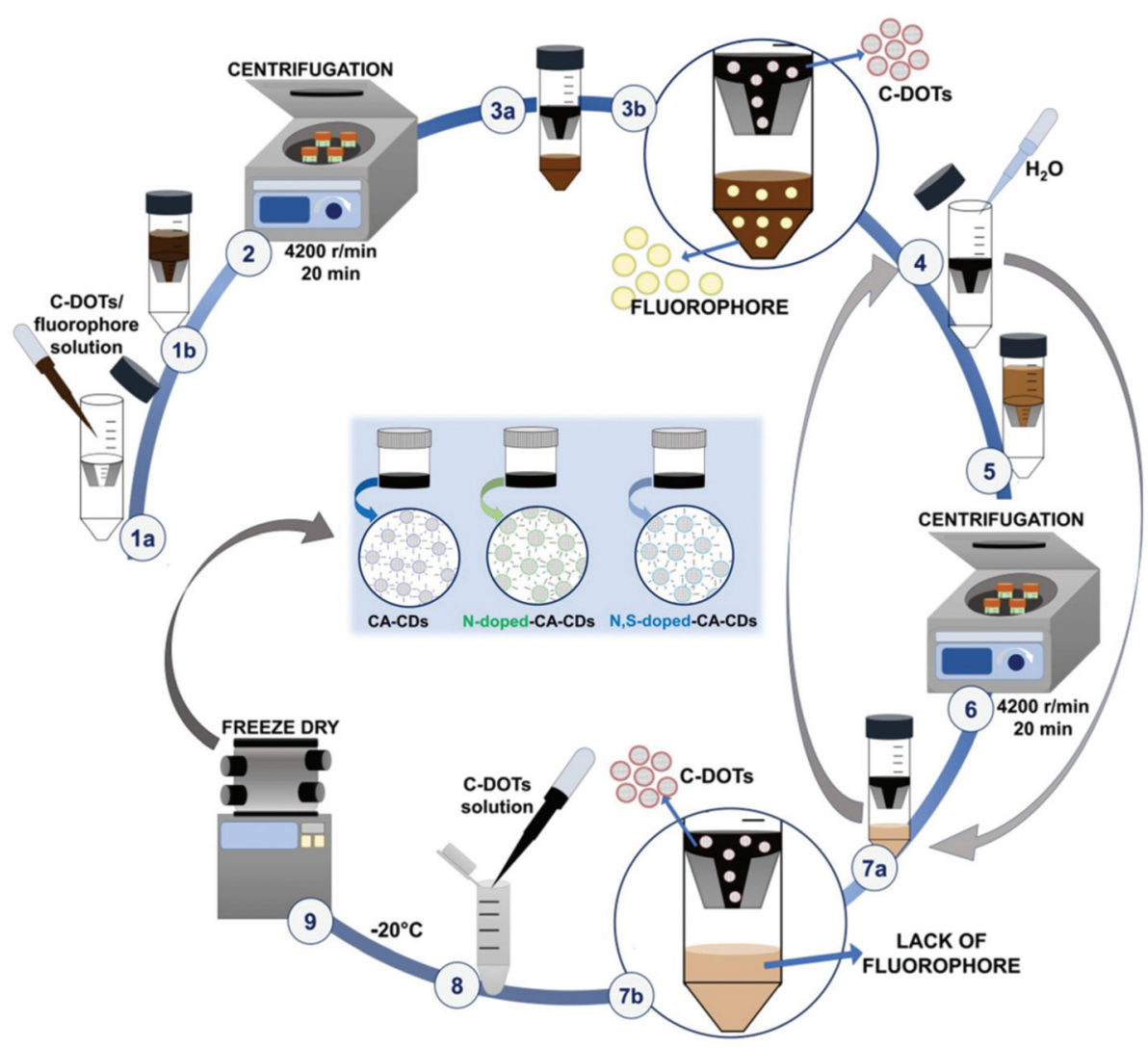

Fig. 3 Carbon dot purification procedure. The process for carbon dot purification was carried out according to the following steps: (1) carbon dot/ fluorophore solution was placed in Vivaspin $₫ 20$ centrifugal concentrators, 10000 MWCO (Sartorius, Germany); (2) the product was centrifuged at a rotation speed of $4200 \mathrm{rpm}$ for 20 minutes; (3) as a result of the centrifugation process, a solution containing carbon dots, the remaining fluorophore and other impurities retained over the membrane, while the low-molecular weight fluorophore fraction passed through it; (4) the fluorophore layer was transferred to another container and distilled water was poured into the concentrator to rinse off the solution from the above membrane; (5) a vial refilled with distilled water was then centrifuged; (6) the filtrate content was analysed again; (7) (a and b) stages 4, 5, 6, 7a, and 7b were repeated until no fluorophore was visible in the filtrate solution; (8) the layer containing pure carbon dots was transferred to a separate pot, frozen and lyophilized. 
of the sample conductivity. All XPS spectra were charge referenced to the unfunctionalized, saturated carbon $(\mathrm{C}-\mathrm{C}) \mathrm{C} 1 \mathrm{~s}$ peak at $284.8 \mathrm{eV}$. The operating pressure in the analytical chamber was less than $3 \times 10^{-9}$ mbar. Deconvolution of spectra was carried out using PHI MultiPak software (v.9.9.0.8). The spectrum background was subtracted using the Shirley method.

\section{Electrochemical properties of carbon dots}

Voltammetry measurements were conducted at $25{ }^{\circ} \mathrm{C}$ using the Electrochemical Analyser M161 and the Electrode Stand M164, from MTM-ANKO, Poland with a standard three-electrode cell. Voltammograms were recorded at a scan rate of $100 \mathrm{mV} \mathrm{s}^{-1}$, unless stated otherwise. The working electrode was a glassy carbon electrode of $3 \mathrm{~mm}$ diameter (Mineral, Poland) and a platinum wire acted as an auxiliary electrode. All potentials were measured against the $\mathrm{Ag} / \mathrm{AgCl}(3 \mathrm{M} \mathrm{KCl})$ electrode, which was placed in a double bridge, filled with $3 \mathrm{M}$ $\mathrm{KCl}$ solution in the upper part and a supporting electrolyte solution in a given solvent in the lower part. Two solutions were divided with a cotton wool plug and separated from the test solution with a dense ceramic frit. The potential of this reference electrode at $25{ }^{\circ} \mathrm{C}$ is $+0.209 \mathrm{~V}$ against the standard hydrogen electrode (SHE). $0.1 \mathrm{M} \mathrm{KCl}$ in distilled water was used as the supporting electrolyte. All measurements were carried out under a dry argon (5.0 Messer) atmosphere.

Standard voltammetry measurements were performed in a multi-cycle mode, with 10 cycles and 2 minutes of electrolyte mixing time between cycles. Additionally, the current background recorded for the electrolyte $(0.1 \mathrm{M} \mathrm{KCl}$ in water) was mathematically subtracted from the voltammetry profiles before the signal analysis. It has been reported that CDs exhibited slow electron transfer due to structural effects such as a low edge plane content and a low specific surface area. Therefore, in order to determine the value of redox potentials on the basis of standard CD voltammograms, measured in aqueous solutions, it is necessary to analyse the first derivatives of the obtained voltammetric profiles. Due to the low intensity of the recorded signals and high noise, the determined values of the redox potentials may be burdened with a large error. Therefore, a ferro/ferricyanide redox probe for comparative purpose was used to investigate the electrochemical properties of the analysed carbon dots. Potassium ferricyanide was used as a probe $\left(E_{\text {ox }}=250 \mathrm{mV}, E_{\text {red }}=180 \mathrm{mV}\right)$. It can be noted that as the concentration of carbon dots increases, the intensity of the signals observed for potassium ferricyanide decreases. By analysing the first derivatives of the voltametric profiles recorded for carbon dots and the change in the intensity of signals recorded for potassium ferricyanide while increasing the concentration of carbon dots, it can be estimated that the oxidation potential of the analysed carbon dots is about $300 \mathrm{mV}$, while the reduction potential is about $-200 \mathrm{mV}$.

\section{Carbon dot spectroscopic characterization}

All the absorption properties of carbon dots including substrates were analysed using a SilverNova TEC-X2 spectrophotometer (StellarNet, Inc., Tampa, FL, USA) in the range of
190-1100 nm. A UV/Vis deuterium/halogen light source (StellarNet, Inc., Tampa, FL, USA) in the range of 190-2500 nm was used in all experiments. Fluorescence emission and excitation spectra of CA-CDs, N-doped-CA-CDs and N,S-dopedCA-CDs were analysed using a FluoroMax-4P spectrofluorometer (Horiba, Kyoto, Japan). All spectra were recorded at varied excitation wavelengths in the range of $200-800 \mathrm{~nm}$, using a slit width equal to $4 \mathrm{~nm}$ in each measurement, both for excitation and emission.

\section{Real-time FT-IR photopolymerization experiments}

The photopolymerization process induced by light with the assistance of carbon dots was analysed by the FT-IR method in real time, using an FT-IR i10 NICOLET ${ }^{\mathrm{TM}}$ infrared spectrometer with a horizontal adapter (Thermo Scientific, Waltham, MA, USA) (Fig. S15 $\dagger$ ). Components for these experiments were prepared by dissolving appropriate amounts of the initiating system with different monomers under no-light conditions. Furthermore, the kinetics measurement included monitoring of absorbance changes at specified wavelengths corresponding to the functional group or binding of the utilized monomers. Therefore, the conversion degree was calculated using the following formula:

$$
\text { Conversion }[\%]=\left(1-\frac{\text { Area }_{\text {After }}}{\text { Area }_{\text {Before }}}\right) \times 100 \%
$$

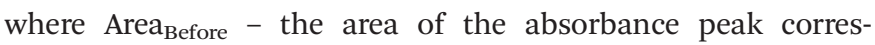
ponding to a given group or bond in the monomer before

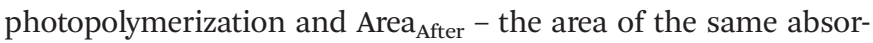
bance peak at a given polymerization time. The wavelengths corresponding to the analysed groups or bonds during the polymerization are presented separately for each of the monomers in the further course of the investigation (Fig. S17 $\dagger$ ).

\section{Cationic photopolymerization of vinyl monomers}

For the light-induced cationic polymerization of vinyl monomers, tri(ethylene glycol) divinyl ether (TEGDVE) was mixed together with the photoinitiating system: appropriate carbon dots/iodonium salt $(0.2 / 2 \mathrm{wt} \%)$ in a mass ratio to monomer content. The measurement was carried out in laminate form for $400 \mathrm{~s}$. The vinyl content was continuously monitored using an infrared spectrometer FT-IR at about $1625 \mathrm{~cm}^{-1}$. As a reference, the TEGDVE monomer with $2.0 \mathrm{wt} \%$ of iodonium salt was used. Additionally, to increase the speed and degree of conversion of the polymerization reaction induced by light of $405 \mathrm{~nm}$, an additive in the form of $N$-vinylcarbazole (2.0 wt\%), under identical measurement conditions as above, was used.

\section{Cationic ring opening photopolymerization process of epoxy monomers (ROP)}

A photocurable composition made of 3,4-epoxycyclohexylmethyl-3,4-epoxycyclohexane-carboxylate (CADE) and an appropriate initiating system, IOD/TTMSS/C-dots (2/2/0.2 wt $\%)$ were prepared for monitoring ring-opening cationic photo-

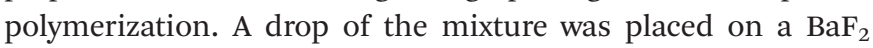
pallet (the thickness of the composition was around $25 \mu \mathrm{m}$ ). 
The epoxy content was monitored using an infrared spectrometer FT-IR at about $790 \mathrm{~cm}^{-1}$ for $800 \mathrm{~s}$. As a reference, the epoxy monomer with $2.0 \mathrm{wt} \%$ of IOD and $2.0 \mathrm{wt} \%$ of TTMSS was used.

\section{Free-radical photopolymerization of acrylate monomers}

A composition made of trimethylolpropane triacrylate TMPTA, together with a two component initiating system IOD ( $2 \mathrm{wt} \%$ ) as a photoinitiator and carbon dots $(0.2 \mathrm{wt} \%)$ as photosensitizers were prepared for the investigation of free-radical photopolymerization. A drop of the mixture was placed between two propylene films to eliminate the oxygen inhibition and then placed on a horizontal holder in an infrared spectrometer FT-IR. The thickness of the composition was around $25 \mu \mathrm{m}$. The content of the double bond was continuously monitored at about $1635 \mathrm{~cm}^{-1}$ for $800 \mathrm{~s}$. As a reference, the TMPTA monomer with $2.0 \mathrm{wt} \%$ of iodonium salt was utilized.

\section{A visible photoinitiator system based on carbon dots for free- radical polymerization of the water-based coating hydrogel under air}

Free-radical photopolymerization of 2-hydroxyethyl acrylate (HEA) in an aqueous environment was performed since carbon dots are known to absorb in a wide range of wavelengths; ${ }^{50}$ therefore, the possibility of using them with different light sources has been investigated. A photocurable composition was prepared using the following materials: carbon dots (0.2 wt $\%)$, bis-(4-t-butylphenyl)iodonium hexafluorophosphate, IOD $(2 \%)$ and a mixture of HEA monomer and water in a $1: 1$ mass ratio. The composition ( $0.5 \mathrm{~mm}$ thick) was applied on a specially prepared barium fluoride adapter (Fig. S16†), allowing the possibility of monitoring the polymerization reaction despite the presence of water. The double bond conversion was followed on an infrared spectrometer FT-IR at about $6125 \mathrm{~cm}^{-1}$. As a reference, a mixture of $\mathrm{HEA} / \mathrm{H}_{2} \mathrm{O}(1: 1)$ with $2.0 \mathrm{wt} \%$ of iodonium salt was used.

\section{Photocatalytic properties of carbon dots during the free- radical polymerization of the water-based coating hydrogel}

The catalytic properties of carbon dots have been confirmed following the free-radical polymerization reaction of the mixture of the HEA monomer and water (1:1) in various systems: I. carbon dots $(0.2 \mathrm{wt} \%)$ and bis-(4- $t$-butylphenyl) iodonium hexafluorophosphate, IOD (2\%), II. carbon dots (0.2 wt\%) and methyl diethanolamine, MDEA (2\%), and III. carbon dots/IOD/MDEA (0.2/2/2 wt $\%)$. The mass ratios of the components were calculated from the quantity of the monomer-water mixture. As a reference, I, II, and III compositions without carbon dots were investigated. The kinetics of the acrylate monomer polymerization process was monitored using a Real-Time infrared spectrometer FT-IR following peak decay at $6125 \mathrm{~cm}^{-1}$ for $300 \mathrm{~s}$.

\section{D-VAT printing of photocurable compositions}

3D-VAT printouts were obtained using a laser printer (DK-8-KZ, NEW) under a laser light source (a405 nm (spot diameter
$50 \mu \mathrm{m})$ with an intensity of $90 \mathrm{~mW} \mathrm{~cm}^{-2}$. VAT photopolymerization is an additive manufacturing process in which the liquid photopolymer in a vat is selectively cured by lightactivated polymerization. For experiments with 3D printing, the photocurable cationic resin was used based on the vinyl monomer (TEGDVE) and contains a photoinitiating system composed of carbon dots as photosensitizers and diphenyliodonium salts (IOD) at a concentration of $0.2 / 2 \mathrm{wt} \%$. All obtained 3D printouts were analysed using the digital microscope DSX1000 with an objective lens DSX10-SXLOB3X with a magnification of 42-420X (Olympus, Japan).

\section{Three-dimensional printing of the hydrogel model structure}

Three-dimensional hydrogels were prepared by visible LED curing of aqueous solutions containing 50\% (w/w) HEA monomer with a photoinitiating system based on CDs and diphenyliodonium salt (IOD) at a concentration of $0.2 / 2 \mathrm{wt} \%$. A predesigned hydrogel model was 3D printed using a laser printer (DK-8-KZ, NEW) under a laser light source @405 nm (spot diameter $50 \mu \mathrm{m}$ ) with an intensity of $90 \mathrm{~mW} \mathrm{~cm}^{-2}$. After $3 \mathrm{D}$ printing, the structures were observed under the digital microscope DSX1000 with an objective lens DSX10-SXLOB3X with a magnification of 42-420X (Olympus, Japan).

\section{Irradiation sources}

For real-time FTIR experiment, the following sources of light were used: a $365 \mathrm{~nm}$ M365LP1 UV-LED diode $\left(I_{0}=24.8 \mathrm{~mW}\right.$ $\mathrm{cm}^{-2}$, Thorlabs, Tampa, FL, USA), a $405 \mathrm{~nm}$ M405LP1 LED diode $\left(I_{0}=22.0 \mathrm{~mW} \mathrm{~cm}{ }^{-2}\right.$, Thorlabs, Tampa, FL, USA), a $415 \mathrm{~nm}$ M415LP1 LED diode $\left(I_{0}=24.0 \mathrm{~mW} \mathrm{~cm}{ }^{-2}\right.$, Thorlabs, Tampa, FL, USA) and a $450 \mathrm{~nm} \mathrm{M450LP1} \mathrm{LED} \mathrm{diode}\left(I_{0}=\right.$ $27.1 \mathrm{~mW} \mathrm{~cm}^{-2}$, Thorlabs, Tampa, FL, USA). All LEDs were powered using a DC2200 controller (Thorlabs, Tampa, FL, USA). The distance between the irradiation sources and sample was $2.1 \mathrm{~cm}$.

\section{Results and discussion}

High-performance technologies require materials that are required for clean production and normal individual applications. ${ }^{51}$ Moreover, environmental and socio-economic sustainability based on new nanotechnologies combines the maximum efficiency with a minimum cost of substrates consumed in photopolymerization processes. ${ }^{52}$ In photocatalytic developments, the employment of carbon dots recently showed their effectiveness and efficiency. ${ }^{47,48}$ Therefore, carbon dots formed as a result of the "bottom-up" condensation reaction of citric acid with amino and sulfuric promoters $^{41-43}$ appear to be a suitable alternative to current photoinitiators, guaranteeing appropriate properties for lightinduced processes.

In this study, we synthesized CDs via "bottom up" condensation from CA alone and using amino and sulphur dopants i.e., urea and thiourea, respectively. The reaction products were then purified to obtain structurally different CDs from citric 
acid containing various dopants including nitrogen and sulphur atoms (ESI†). The bottom-up approach for the fabrication of these materials needs to be carefully examined in terms of possible interactions of the dopant with citric acid. Therefore, a detailed analysis of the purification process of the synthesized CDs was conducted. Purification of the CDs is essential for removing free low-molecular compounds, ligands, or other substrates potentially triggering side effects. For this reason, we used the ultrafiltration process to purify, concentrate and separate CDs from the substrates and other small organic molecules. This study showed that CDs were treated by ultrafiltration using Vivaspin ${ }^{\circledR} 20$ centrifugal concentrator twin vertical membranes. Due to the vertical construction of the membrane, high flow and recovery values were achieved. Noteworthy is that purification and concentration steps were performed simultaneously.

\section{Determination of the chemical bonding and chemical composition of carbon dots}

Surface concentrations of chemical bonds obtained from fitting XPS data for all analyzed samples are listed in Table 1. The C 1s spectra for all samples can be fitted with five components: the first line at $284.0 \mathrm{eV}$ arising from $\mathrm{C}=\mathrm{C}$ type bonds $\left(\mathrm{sp}^{2}\right)$, the second line at $284.8 \mathrm{eV}$ originating from $\mathrm{C}-\mathrm{C}$ type carbon bonds $\left(\mathrm{sp}^{3}\right)$, the third line at $286.6 \mathrm{eV}$ from $\mathrm{C}-\mathrm{O}-\mathrm{C}$ and/or $\mathrm{C}-\mathrm{OH}$ and/or C-NH bonds, the fourth line at $288.3 \mathrm{eV}$ coming from $\mathrm{C}=\mathrm{O}$ and/or $\mathrm{O}-\mathrm{C}-\mathrm{O}$ and/or $\mathrm{N}-\mathrm{C}-\mathrm{O}$ bonds and the last line centered at $289.1 \mathrm{eV}$ from the $\mathrm{O}-(\mathrm{C}-\mathrm{O})-\mathrm{O}$ and/or $\mathrm{N}-\mathrm{C}=\mathrm{O}$ and/or shake-up satellite from aromatic-type rings. ${ }^{53}$ Spectra collected for all samples in the $\mathrm{N} 1 \mathrm{~s}$ region show a single peak centered at $399.7 \mathrm{eV}$ which originates from the imine $\mathrm{C}=\mathrm{N}-\mathrm{R}$ and/or $\mathrm{CH} 2-\mathrm{NH} 2$ and/or pyridine form and/or imide $(\mathrm{C}=\mathrm{O})-\mathrm{N}-(\mathrm{C}=\mathrm{O})$ and/or $-\mathrm{NH}-(\mathrm{C}=\mathrm{O})-\mathrm{O}$ or $-\mathrm{NH}-$ attached to the aromatic ring. ${ }^{53,54}$ Spectra collected in the $\mathrm{O} 1 \mathrm{~s}$ region are similar for all samples and can be fitted with two lines centered at $531.2 \mathrm{eV}$ and $532.8 \mathrm{eV}$. The first peak can be attributed to $\mathrm{O}=\mathrm{C}-\mathrm{N}$ and/or $\mathrm{C}=\mathrm{O}$ bonds, whereas the second to $\mathrm{O}=\mathrm{C}-\mathrm{O}^{*}$ and/or $\mathrm{O}-\mathrm{C}-\mathrm{O}$ and/or C-OH-type bonds. ${ }^{53,54}$ The $\mathrm{S}$ $2 \mathrm{p}$ spectra for all samples show a doublet structure (doublet separation $\mathrm{p} 3 / 2-\mathrm{p} 1 / 2$ equals $1.2 \mathrm{eV}$ ) with the main line $2 \mathrm{p} 3 / 2$ centered at $163.4 \mathrm{eV}$ which indicates either $\mathrm{C}-\mathrm{S}-\mathrm{C}$ sulfide or C-SH thiol-type bonds. ${ }^{55,56}$ Sodium was found on all samples and the corresponding $\mathrm{Na}$ 1s spectra were fitted with the single line centered at $1071.1 \mathrm{eV}$ which indicates the presence of $\mathrm{Na}^{+}$ions in either $\mathrm{NaOH}$ or oxides and/or sub-oxides or its salts. ${ }^{56}$ The result of XPS analysis is shown in detail in the ESI (Fig. S6-S11†).

Furthermore, the morphology of the CDs was imaged by transmission electron microscopy: the quantum dots were irregular in appearance $\left(\Phi_{\text {avg }}=10 \pm 5 \mathrm{~nm}\right.$; Fig. $\left.4 \mathrm{~b}\right)$.

High quality TEM images are difficult to obtain, because the samples at the final stage of synthesis were lyophilized and then they probably aggregated. Therefore, the size of the obtained CDs in aqueous solution was additionally measured by dynamic light scattering. The obtained results confirm that the sizes of the obtained CDs vary around $10 \mathrm{~nm}$. TEM ana-

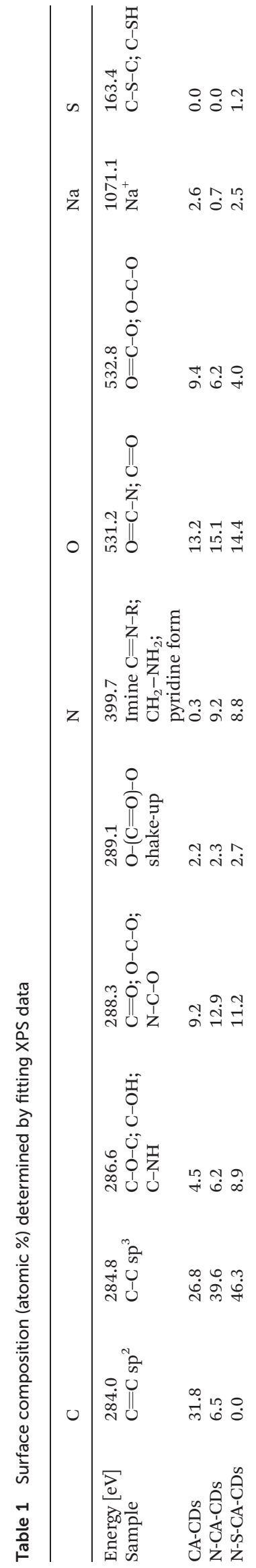



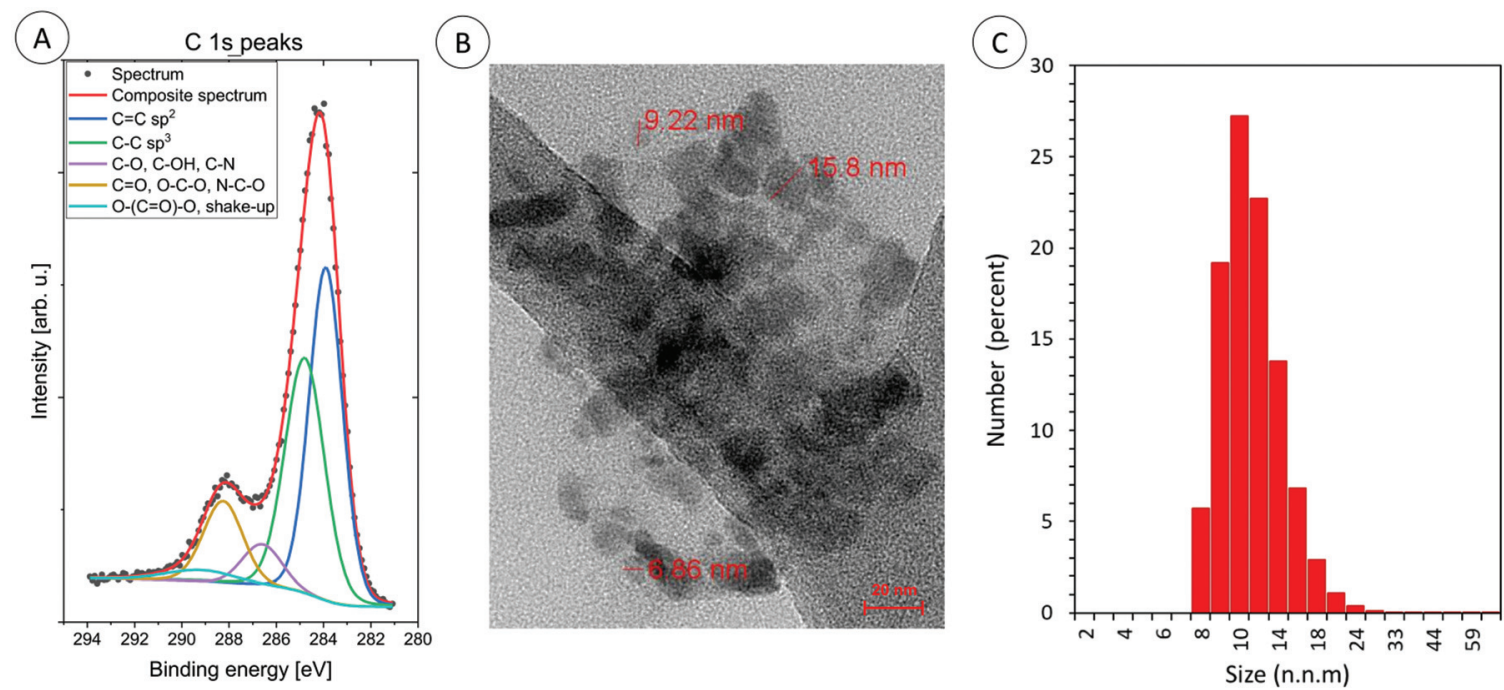

Fig. 4 (A) The high energy resolution XPS spectra of $C$ 1s peaks for carbon dots: CA-CDs, (B) TEM image of carbon dots: CA-CDs, and (C) DLS particle size distribution histogram of CA-CDs.

lysis, together with XPS and DLS analysis is exemplified for CA-CDs in Fig. 4.

The size distribution and the average size of the prepared carbon dots were directly determined in the aqueous suspension (0.1 $\mathrm{mg} \mathrm{mL}^{-1}$ ) using dynamic light scattering (DLS) (Zetasizer Nano ZS instrument from Malvern). Due to significant aggregation of CDs at neutral $\mathrm{pH}$, all measurements were conducted at $\mathrm{pH} 10$ ( $\mathrm{NaOH}$ was used for $\mathrm{pH}$ adjustment) (Fig. S12-S14†).

\section{Determination of the spectroscopic properties of carbon dots}

In order to determine the spectroscopic properties of the monitored carbon dots, their absorbance and fluorescence using water as a solvent were investigated. The absorbing properties of carbon dots are characteristic of this group of materials ${ }^{57}$ exhibiting strong light absorption in the UV region with a tail extending into the visible range reaching above $800 \mathrm{~nm}$. The maximum absorption peak in the UV-C range may correspond to a $\pi-\pi^{*}$ transition involving aromatic $\mathrm{sp}^{2}$ carbons, and the tail starting from $300 \mathrm{~nm}$ can be assigned to an $n-\pi^{*}$ transition involving functional groups with electron lone pairs. The red-shifted absorption spectrum is directly attributed to the addition of a heteroatom e.g., nitrogen, into the carbon $\mathrm{sp}^{2}$ lattice because the nitrogen centres can inject excess electrons into the unoccupied $\pi^{*}$ orbitals and markedly reduce the HOMO-LUMO gap and the energies of the corresponding optical transitions. ${ }^{58}$ The visible absorption of CDs make them perfect materials for photocatalytic applications. For comparison, the absorbance of carbon dots was compared with the absorbance of substrates used in their synthesis in Fig. 5 .

The photographs of CA-CD, N-doped-CD and N,S-doped-CD aqueous solutions exposed to different conditions are presented in Fig. 6: in daylight as well as with light exposure at

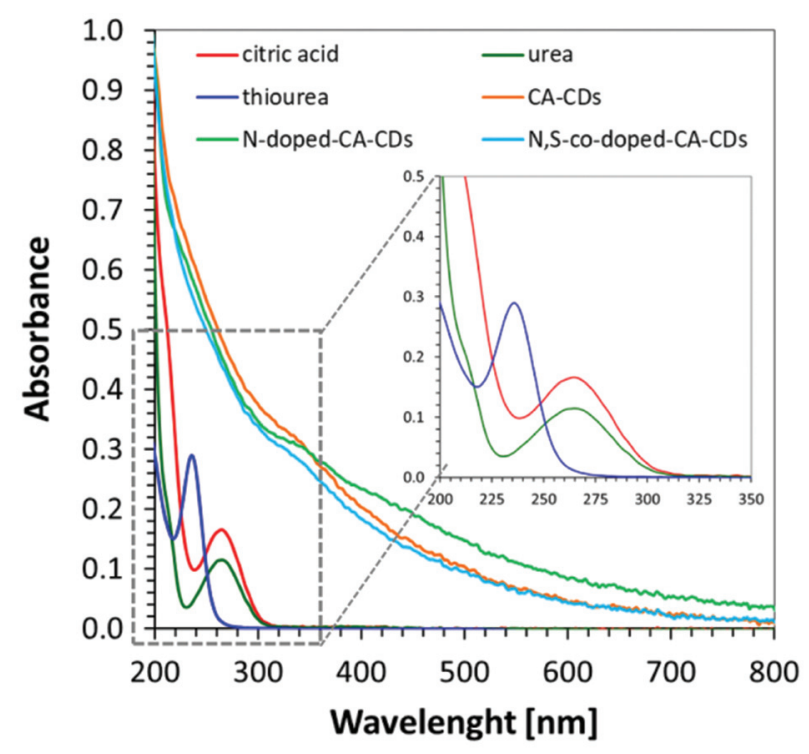

Fig. 5 UV-visible absorption spectra of the synthesized citric acidbased CDs (CA-CDs, N-doped-CA-CDs, N,S-Co-doped-CA-CDs) dissolved in water $\left(0.01 \mathrm{~g} \mathrm{~mL}^{-1}\right)$ and the organic molecular precursors for the synthesis of CA-CDs.

$254 \mathrm{~nm}$ and $365 \mathrm{~nm}$. When using light at $365 \mathrm{~nm}$, the aqueous solutions of carbon dots showed emission from blue to green luminescence, which was the most intense for carbon dots enriched with nitrogen and sulphur dopants (Fig. 6). Moreover, carbon dots are characterized by controllable photoluminescence, and their emission characteristics have a direct correlation with the excitation wavelength. ${ }^{59}$ Therefore, we have examined the emissions of the synthesized carbon dots using different excitation wavelengths in the range from approximately $400 \mathrm{~nm}$ to $500 \mathrm{~nm}$. The results of the performed studies are presented in Fig. 6. 

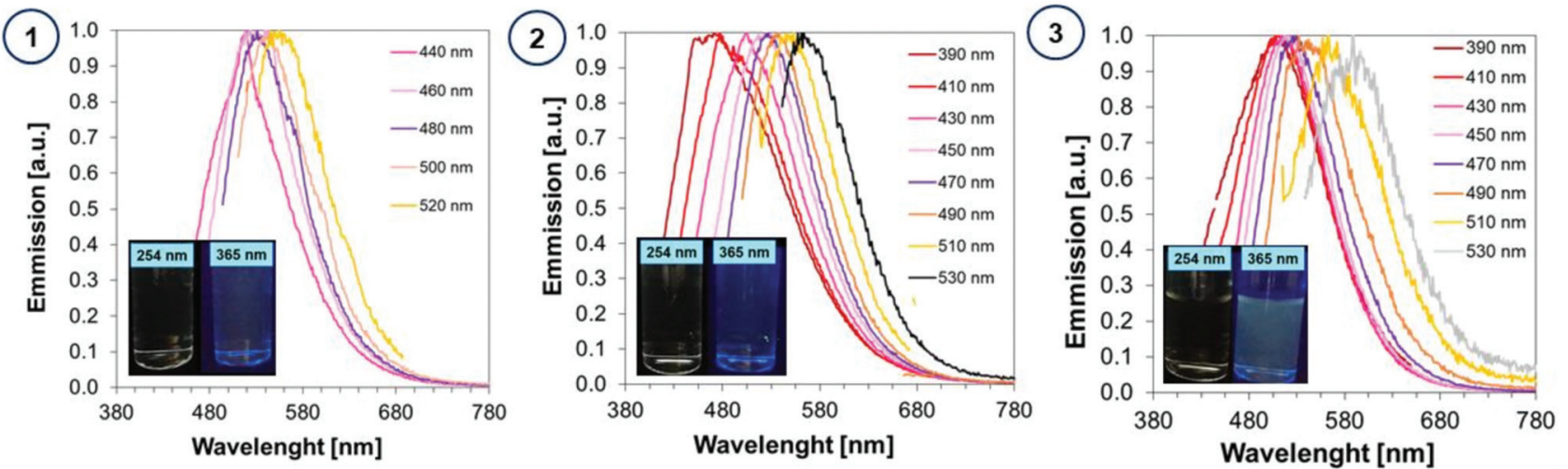

Fig. 6 Fluorescence spectra of aqueous solutions of (1) CA-CDs, (2) N-doped-CA-CDs, and (3) N,S-doped-CA-CDs determined with the use of various excitation wavelengths.

\section{Real-time FT-IR experiments}

Cationic photopolymerization of the vinyl monomer. Carbon dots among nanomaterials in the field of polymer chemistry and photopolymerization processes are well known. Nevertheless, their application focuses exclusively on freeradical polymerization, including in particular the RAFT or ATRP reaction, as proven by scientific reports. ${ }^{47-49}$ Herein, for the first time, we propose carbon dots for use as green photosensitizers of iodonium salt in cationic photopolymerization processes with the chain growth mechanism including ring opening photopolymerization (ROP).

However, the spectroscopic properties of currently used commercial one component cationic photopolymerization initiators do not match the emission range of lamps used as the basic light sources in the photochemical industry. There is only a slight overlap between the absorption and emission bands. This makes the initiation process very inefficient and most of the electrical energy supplied to the lamps is wasted. Hence, the development of new cationic photoinitiators, which require shifting their absorption characteristics to longer wavelengths with increased efficiency of cationic photopolymerization processes, is a major industrial problem. Another important aspect in the exploration for new cationic photoinitiators is the reduction of the environmental impact of polymerization processes. ${ }^{60}$ Thus, the development of highly efficient cationic photoinitiators for visible light polymerization has become an extensive study focusing on synthesis through structural modifications and improvement of spectroscopic properties, as well as their application in the rapidly growing 3D printing. For this reason, this paper focused on the fundamental study of new multicomponent cationic photoinitiating systems composed of iodonium salts and appropriate bio-based photosensitizers in the form of CDs, acting via a photoinduced electron transfer process. The first step was to use CDs as a photosensitizer of iodonium salts during the chain growth cationic polymerization of the vinyl monomer TEGDVE. Considering that CDs are nanomaterials sensitive to visible light, this study has been undertaken to reveal their ability to initiate the polymerization of new photoinitiating systems based on carbon dots and iodonium salts as bimolecular photoinitiating systems $(2 / 0.2 \mathrm{wt} \%$ in relation to the weight of the used monomer). As a reference, a mixture of TEGDVE monomer and iodonium salts (2 wt\%) was used. The reactions were carried out in air. Conversion-time profiles using two light sources: UV-LED @365 nm and Vis-LED (a) $405 \mathrm{~nm}$ are shown in Fig. 7, while the obtained conversion degrees are listed in Table 2.

The proposed initiating system IOD/CDs effectively initiates the process of the cationic photopolymerization of the vinyl monomer TEGDVE using UV-A light, guaranteeing relatively high degrees of monomer conversion. Disturbing kinetic curves were obtained while using light from the visible range (LED @405 nm), where the proposed initiating system proved to be insufficient. Therefore, it was proposed to use an accelerating additive in the form of $N$-vinylcarbazole, when exposed to light at $405 \mathrm{~nm}$.

The time-conversion relationship for a system containing diaryliodonium salt (IOD) $(2.0 \quad \mathrm{wt} \%), \quad N$-vinylcarbazole $(2.0 \mathrm{wt} \%)$ and $\mathrm{N}, \mathrm{S}$-doped-CA-CDs $(0.2 \mathrm{wt} \%)$ is shown in Fig. 8 together with the scheme of the initiation photocatalytic cycle involving CA-CDs as photocatalysts for cationic polymerization in an oxidative process at a low light intensity. The proposed solution to the problem caused by the use of light from the visible range has been effectively proved. The use of $N$-vinylcarbazole accelerates the IOD/CD initiating system and thus provides a high degree of conversion as well as an increased rate of the polymerization reaction (increase in curve slope). ${ }^{8,61,62}$

Cationic photopolymerization of the epoxy monomer. Accordingly, the possibility of application of carbon dots as sensitizers for the ring opening cationic photopolymerization of the epoxy monomer CADE was investigated. Therefore, we decided to study the effectiveness of the developed IOD/CD initiating systems in light-initiated polymerization of epoxy monomers. For this kind of polymerization, we chose the initiating system supported by a silane additive: IOD (2.0 wt\%), TTMSS $(2.0 \mathrm{wt} \%)$ and various carbon dots $(0.2 \%)$ calculated in 
(a)

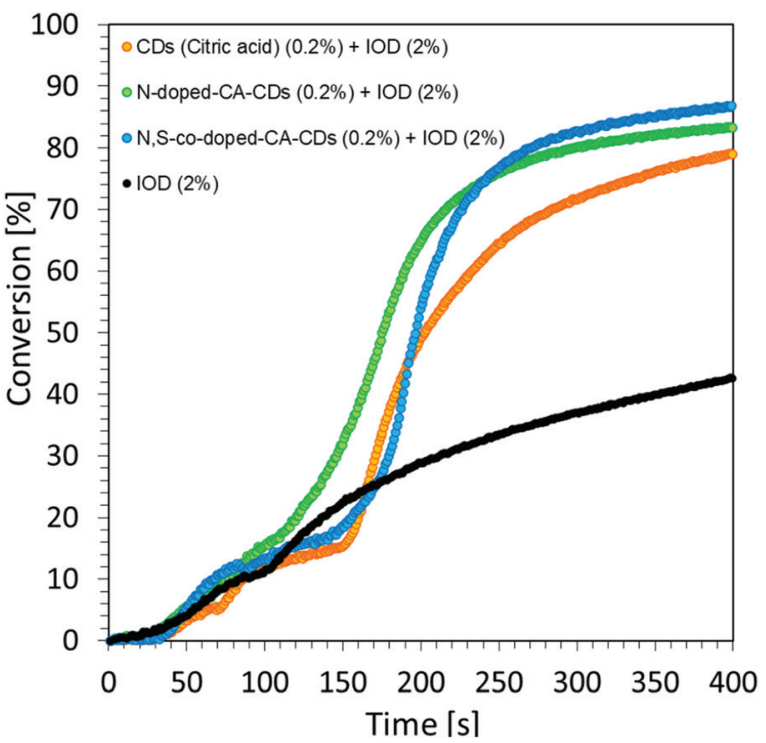

(b)

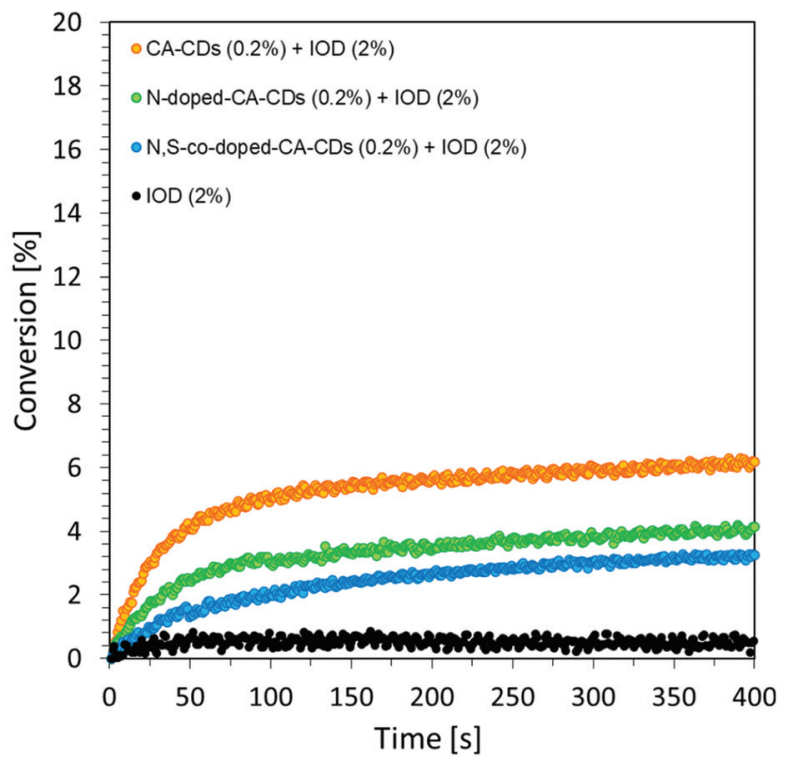

Fig. 7 Cationic photopolymerization profiles (vinyl function conversion vs. irradiation time) initiated by a bimolecular photoinitiating system based on IOD (2.0 wt\%) and CDs (0.2 wt\%) (a) under irradiation at $365 \mathrm{~nm}$ and (b) under irradiation at $405 \mathrm{~nm}$. relation to the content of the epoxy monomer CADE. As a source of light an LED from the safe UV range @365 nm was used, and the reactions were carried out in air for $800 \mathrm{~s}$. The dependence of the conversion on time is shown in Fig. 9, and a summary of the obtained conversion degrees is given in Table 1. The TTMSS radical has been characterized for applications in photoinitiation systems due to a high inherent activity for the addition to double bonds and the ionization potential which is associated with an oxidation process and the formation of silylium cations. The photoinitiator systems based on TTMSS exhibited a high reactivity in free-radical promoted cationic polymerization (FRPCP). Moreover, the ability to efficiently consume oxygen is a particularly interesting feature of the photoinitiating system (PIS) based on TTMSS. ${ }^{63}$ From the obtained results, it is clear that the proposed threecomponent initiating system containing carbon dots based on the precursor of citric acid successfully initiates the reaction of ring opening cationic photopolymerization of epoxide monomers. In this type of reaction, non-doped carbon dots proved to be the most effective, ensuring a high reaction speed, minimum induction time and sufficient conversion of the epoxy monomer. Although we did not optimize the procedures to obtain the highest possible conversion rates, this study provides a new perspective on the application of CDs in cationic polymerization processes; therefore, taking into consideration the variety of possible carbon dots, additional research on the optimization of the process and structure of CDs will be carried out in order to develop highly efficient photoinitiators. As kinetic research has proven, the investigated carbon dots can be applied as photosensitizers for both the free-radical photopolymerization of acrylate monomers and the cationic photopolymerization of vinyl and epoxy monomers. In these processes, the carbon dots initiate the electron transfer reaction from the photo-excited state through an oxidation process in conjunction with iodine salt (IOD). Due to their excellent spectroscopic properties and ability to absorb light from almost the entire spectral range, carbon dots can be easily oxidized in this process. In turn, IOD shows strong electronaccepting properties and as a result electron transfer is reduced, and the radicals formed in this process then decompose into secondary radicals, which undergo further reactions. Another mechanism that allows initiating the polymerization reaction is the photo-reduction mechanism. In this case, the process of electron transfer takes place from the molecule

Table 2 Summary of the final functional group conversions of different monomers polymerized in cationic photopolymerization processes, with the use of carbon dots and IOD as the photoinitiating system (PIS)

Conversion [\%]

\begin{tabular}{llll}
\hline & $\begin{array}{l}\text { EPOXY monomer } \\
\text { (PIS with TTMSS) }\end{array}$ & $\begin{array}{l}\text { VINYL monomer } \\
\text { (PIS without NVK) }\end{array}$ & $\begin{array}{l}\text { VINYL monomer } \\
\text { (PIS without NVK) } \\
\text { @4405 nm }\end{array}$ \\
\hline CA-CDs & @405 nm & @365 nm & 6 \\
N-Doped-CA-CDs & 42 & 79 & 4 \\
N,S-Doped-CA-CDs & 21 & 83 & 3
\end{tabular}



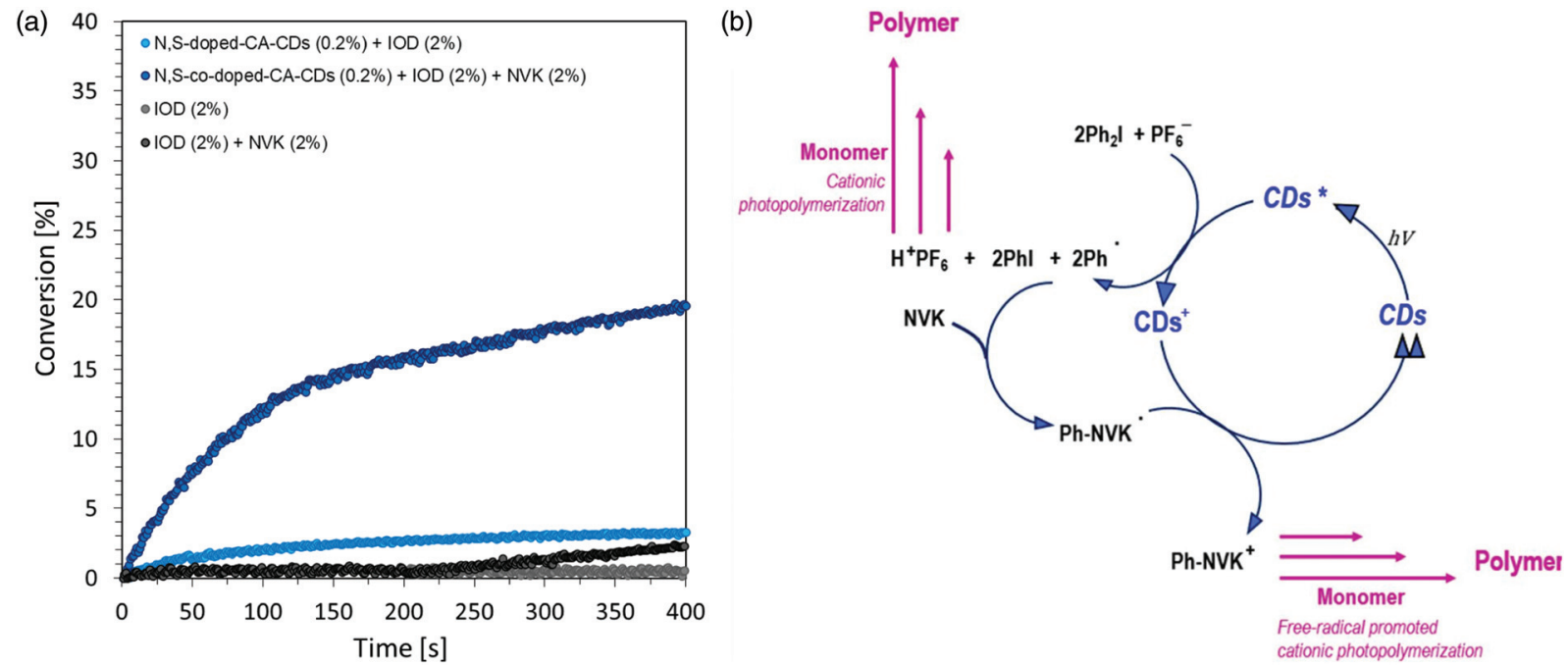

Fig. 8 (a) Cationic photopolymerization profiles (vinyl function conversion vs. irradiation time) initiated by a three-component photoinitiating system based on IOD (2.0 wt\%), NVK (2.0 wt\%) and CA-CDs (0.2 wt\%) under irradiation at $405 \mathrm{~nm}$. (b) Catalytic cycle involving CA-CDs as photocatalysts for polymerization in an oxidative process under low light intensity.
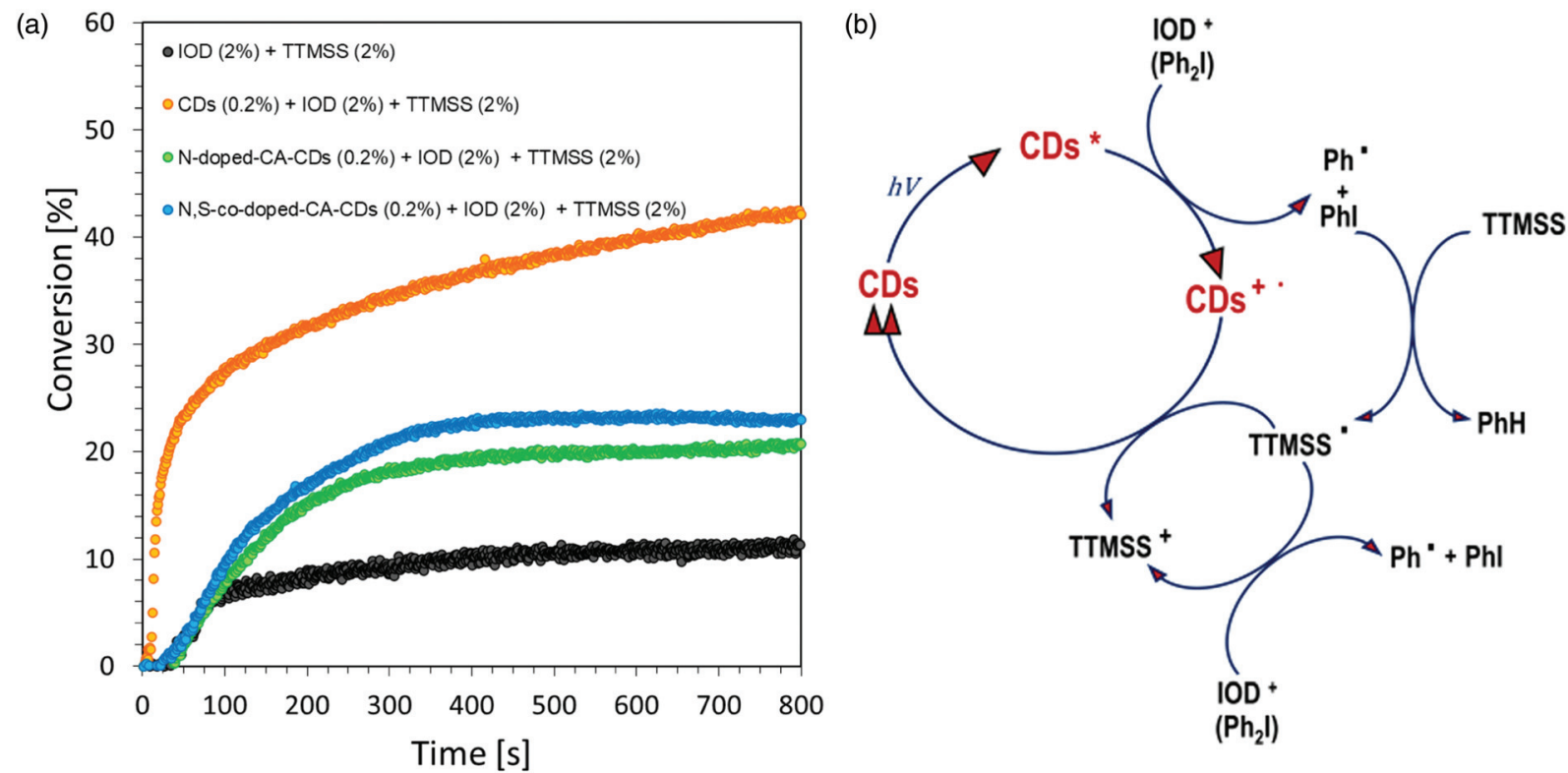

Fig. 9 (a) Cationic photopolymerization profiles (epoxy function conversion vs. irradiation time) initiated by a photoinitiating system based on IOD (2.0 wt\%), TTMSS (2.0 wt\%) and CDs (0.2 wt\%) under irradiation at $405 \mathrm{~nm}$. (b) Schematic cycle of operation of the initiating system consisting of IOD, TTMSS and CDs.

called the co-initiator, for example amines, to the excited individual in this case carbon dots. The analysis of the initiation process according to the mechanism of photo-oxidation and photo-reduction was carried out by examining the oxidation and reduction potentials using cyclic voltammetry and by calculating thermodynamic data for these processes. The obtained results are presented in Table 3.

Changes in the free energy of electron transfer are an important parameter $\left(\Delta G_{\text {et }}\right)$ between the individual components of the photoinitiating system, which requires a nega- tive value for the thermodynamically permitted processes. This value can predict the CDs' potential applicability as the component of the photoinitiator system. The calculation could obtain the electron transfer $(\Delta G)$ value from the $\Delta G_{\text {et }}$ equation. The values of $E_{\mathrm{Ox}}$ and $E_{\text {red }}$ are determined by cyclic voltammetry (Fig. S20-S23 $\dagger$ ). The excited singlet state energy, $E_{\mathrm{S} 1}$, could be calculated by the intersection of normalized absorption and emission spectra (Fig. S19†). It has been reported that CDs exhibited slow electron transfer due to structural effects such as low edge plane content and low specific surface area. 
Table 3 Electrochemical and thermodynamic properties of CA-CDs, N-doped-CA-CDs, and N,S-doped-CA-CDs in the photo-oxidation and photo-reduction mechanisms

\begin{tabular}{|c|c|c|c|c|c|}
\hline Acronym & $E_{\mathrm{S} 1}{ }^{*}[\mathrm{eV}]$ & Estimated $E_{\mathrm{Ox}} v s . \mathrm{Ag} / \mathrm{AgCl}[\mathrm{mV}]$ & $\Delta G_{\mathrm{et}(\mathrm{ox})}^{a}[\mathrm{eV}]$ & Estimated $E_{\text {red }} v s . \mathrm{Ag} / \mathrm{AgCl}[\mathrm{mV}]$ & $\Delta G_{\mathrm{et}(\mathrm{red})}^{b}[\mathrm{eV}]$ \\
\hline N-Doped-CA-CDs & 2.65 & 300 & -1.71 & -200 & -1.59 \\
\hline
\end{tabular}

${ }^{a}$ Calculated from the classical Rehm-Weller equation:

$$
\Delta G_{\mathrm{et}}=F\left[E_{\mathrm{ox}}\left(\mathrm{D} / \mathrm{D}^{\cdot+}\right)-E_{\mathrm{red}}\left(\mathrm{A}^{\cdot-} / \mathrm{A}\right)\right]-E_{\mathrm{s} 1}-\left(\frac{N_{\mathrm{A}} e^{2}}{4 \pi \varepsilon_{0} \varepsilon_{\mathrm{r}} a}\right)
$$

$E_{\mathrm{ox}}\left(\mathrm{D} / \mathrm{D}^{\cdot+}\right)$ - electrochemically determined oxidation potential of the electron donor. $E_{\text {red }}\left(\mathrm{A}^{\cdot-} / \mathrm{A}\right)$ - electrochemically determined reduction potential of the electron acceptor $(-0.64 \mathrm{~V}$ for the diphenyliodonium salt $v s . \mathrm{Ag} / \mathrm{AgCl}) .{ }^{64,65} E_{\mathrm{S} 1}-$ singlet state energy of the sensitiser determined based on the excitation and emission spectra, while using an excitation at a wavelength of $405 \mathrm{~nm}$ (in accordance with the wavelength of light used in the majority of kinetic studies). ${ }^{b}$ Calculated from the equation:

$$
\Delta G_{\mathrm{et}}=F\left[E_{\mathrm{ox}}\left(\mathrm{D} / \mathrm{D}^{\cdot+}\right)-E_{\mathrm{red}}\left(\mathrm{A}^{\cdot-} / \mathrm{A}\right)\right]-E_{\mathrm{S} 1}-\left(\frac{N_{\mathrm{A}} e^{2}}{4 \pi \varepsilon_{0} \varepsilon_{\mathrm{r}} a}\right)
$$

$E_{\mathrm{ox}}\left(\mathrm{D} / \mathrm{D}^{\cdot+}\right)$ - electrochemically determined oxidation potential of the electron donor $(-1.058 \mathrm{~V}$ for amine $\mathrm{EDB} v s . \mathrm{Ag} / \mathrm{AgCl}){ }^{66} E_{\mathrm{red}}\left(\mathrm{A}^{\cdot-} / \mathrm{A}\right)-\mathrm{electro}-$ chemically determined reduction potential of the electron acceptor. $E_{\mathrm{S} 1}-$ singlet state energy of the sensitiser determined based on excitation and emission spectra, while using an excitation at a wavelength of $405 \mathrm{~nm}$ (in accordance with the wavelength of light used in the majority of kinetic studies).

Therefore, in order to determine the value of redox potentials on the basis of standard CD voltamperograms, measured in aqueous solutions, it is necessary to analyze the first derivatives of the obtained voltammetry profiles. Due to the low intensity of the recorded signals and high noise, the determined values of the redox potentials may be burdened with a large error. Therefore, a ferro/ferricyanide redox probe for comparative purpose was used to investigate the electrochemical properties of the analysed carbon dots. Potassium ferricyanide was used as a probe $\left(E_{\mathrm{ox}}=250 \mathrm{mV}, E_{\mathrm{red}}=180 \mathrm{mV}\right)$. It can be noted that as the concentration of carbon dots increases, the intensity of the signals observed for potassium ferricyanide decreases. By analysing the first derivatives of the voltammetric profiles recorded for carbon dots and the change in the intensity of signals recorded for potassium ferricyanide while increasing the concentration of carbon dots, it can be estimated that the oxidation potential of the analysed carbon dots is about $300 \mathrm{mV}$, while the reduction potential is about $-200 \mathrm{mV}$. The $E_{\text {red }}$, and $E_{\mathrm{S}}$ values of CDs are summarized in Table 3 . The presented calculations clearly show that the electron transfer process, according to both the photo-oxidation and photo-reduction mechanisms, is thermodynamically allowed $-\Delta G_{\text {et }}$ values are negative.

Free-radical photopolymerization of acrylate monomers. Free-radical photopolymerization is one of the most popular processes of obtaining polymeric materials, guaranteeing the speed of photopolymerization, control over the kinetics of the process or adjustment to a wide group of monomers based on acrylate or methacrylate. ${ }^{67,68}$ In addition, this type of polymerization is highly tolerant to the presence of water in the system, which makes the radical photopolymerization reaction widely applicable on an industrial scale, not only using the classic method of polymerization, but also using the emulsification or suspension technique. ${ }^{69-71}$ The use of various types of carbon dots for free-radical photopolymerization was first described by Jiang and others, ${ }^{47}$ and they used carbon dots as photosensitizers in the RAFT (Reversible AdditionFragmentation Chain Transfer) polymerization process. Later, Kutahya and others used carbon dots as a "green photocatalyst" in the radical polymerization process, using them in a photocatalytic system based on iodonium or sulphonium salt as a coinitator and carbon dots as a photosensitizer. ${ }^{48}$ However, for the effective operation of such a system, the use of catalytic amounts of $\mathrm{Cu}^{\mathrm{II}}$ is necessary. Therefore, in this perspective, this system cannot be fully reliant on green systems. Our group has attempted to develop a system that does not require an additional component in the form of a transition metal, thus creating more "eco-friendly" conditions. Therefore, we proposed a system consisting of carbon dots $(0.2 \mathrm{wt} \%)$ and iodonium salt (IOD $2.0 \mathrm{wt} \%$ ) to initiate the free-radical photopolymerization reaction of the acrylic monomer TMPTA. As a reference, we used a mixture of TMPTA monomer and iodonium salt $(2.0 \mathrm{wt} \%)$. The reaction was conducted under oxygen-free conditions for $800 \mathrm{~s}$, using two types of light: UV-LED @365 nm and Vis-LED @405 nm, respectively. Conversion-time profiles for both sources of light are given in Fig. 10. The results clearly show that the proposed bimolecular photoinitiating systems based on carbon dots and iodonium salt effectively initiate the free-radical polymerization reactions of an acrylate monomer TMPTA. The doping of carbon dots with heteroatoms significantly affects the degree of conversion of the monomer, but does not affect the induction time or the slope of the kinetic curve. The best results were obtained with nitrogen and sulphur-doped carbon dots for both light sources.

Regardless of the type of photosensitizer used, satisfactory degrees of conversion were obtained without the use of a transition metal as a catalyst for the process. The list of conversion 
(a)

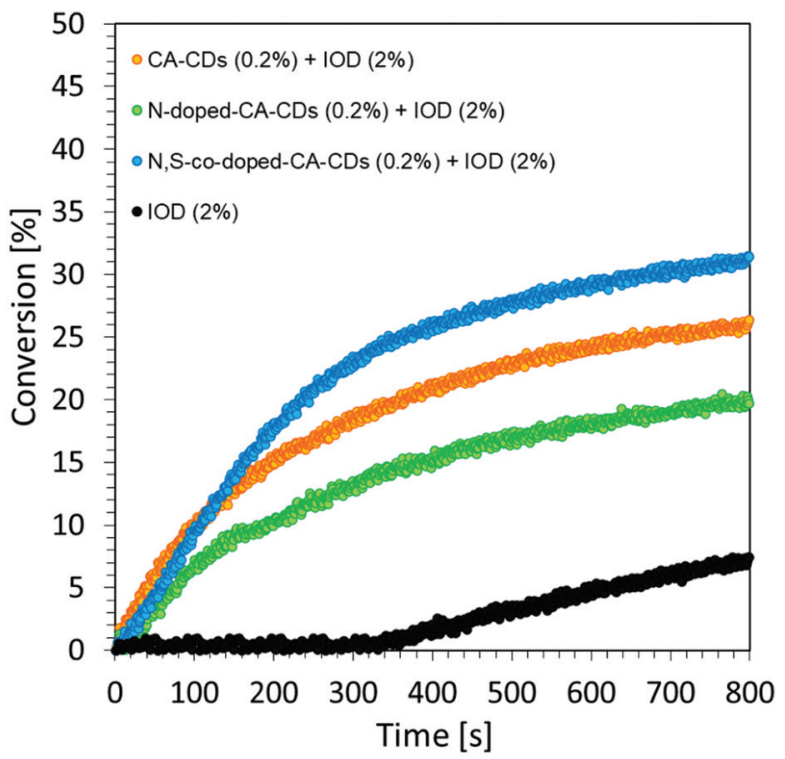

(b)

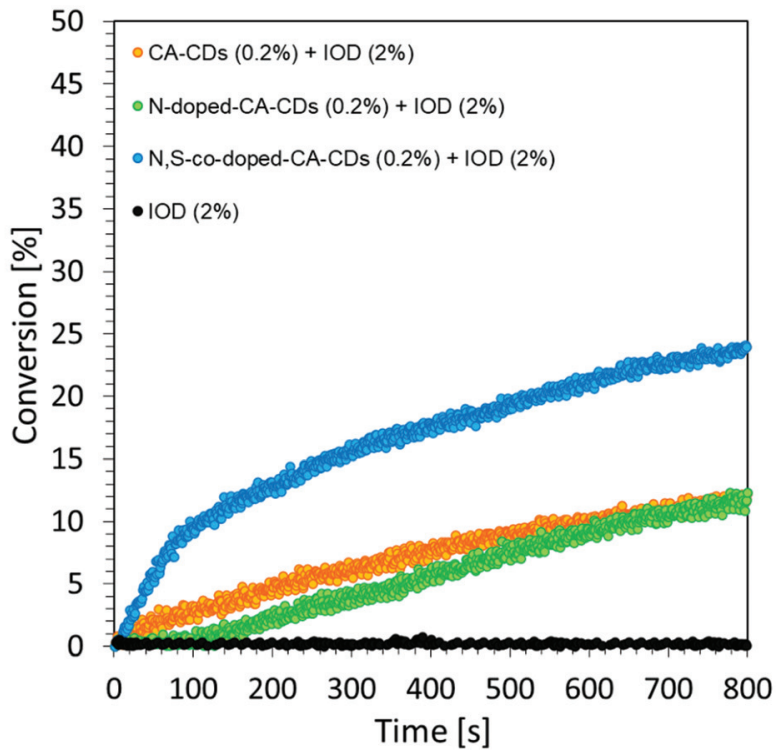

Fig. 10 Free-radical photopolymerization profiles (acrylate function conversion vs. irradiation time) for TMPTA with the bimolecular photoinitiating system based on IOD (2.0 wt\%) and CDs (0.2 wt\%) (a) under irradiation at $365 \mathrm{~nm}$; (b) under irradiation at $405 \mathrm{~nm}$. Irradiance was measured at a distance of $210 \mathrm{~mm}$ for all sources.

rates obtained during the photopolymerization of the acrylic monomer is presented in Table S2. $\dagger$

Free-radical photopolymerization processes of acrylate-water resin - formation of hydrogels. As mentioned in the above considerations, free radical polymerization is insensitive to the presence of water, which makes it possible to use it to obtain hydrogel materials. Hydrogels are extremely interesting in terms of biomedical applications, due to the fact that their structural and biochemical properties are similar to the extracellular matrix (ECM) of most tissues. ${ }^{72}$ In addition, they have a high porosity, which ensures high permeability to nutrients, oxygen and metabolic products. ${ }^{73}$ Most frequently, watersoluble monomers of synthetic origin, e.g., polyethylene glycol or methacrylate or acrylate monomers, e.g., 2-hydroxyethyl acrylate $\mathrm{HEA}^{74}$ or of natural origin $e . g$. chitosan $^{75}$ or collagen ${ }^{76}$ were used in this context. The promising properties of hydrogel materials make it an attractive research topic for scientists around the world, especially in the field of developing new initiators and initiating systems. In this case, the use of carbon dots as sensitizers in the initiation process is particularly justified - they are characterized by good solubility in water, they work effectively with iodonium salt and are derived from natural, non-toxic sources. In addition, their characteristic absorption covering a broad wavelength range makes carbon dots a promising candidate as photosensitizers with the use of safe sources of light in the visible range.

Carbon dots as effective photosensitizers at visible wavelengths under oxygen conditions and in an aquatic environment. CDs present a broad absorption spectrum that spreads from the UV to the red region. Therefore, PISs based on CDs as photosensitizers could be an efficient initiating system that would ensure polychromatic light excitation (thereby allowing the collection of a large number of visible photons) or selected monochromatic excitations delivered by different sources of LEDs. To the best of our knowledge, the ability of CDs to be involved in panchromatic PISs was never investigated. The ability of CDs to act as free-radical photoinitiator systems is exemplified by the conversion curves in Fig. 11 and Table 4, which show the values of rates of conversion and final conversions at the different wavelengths.

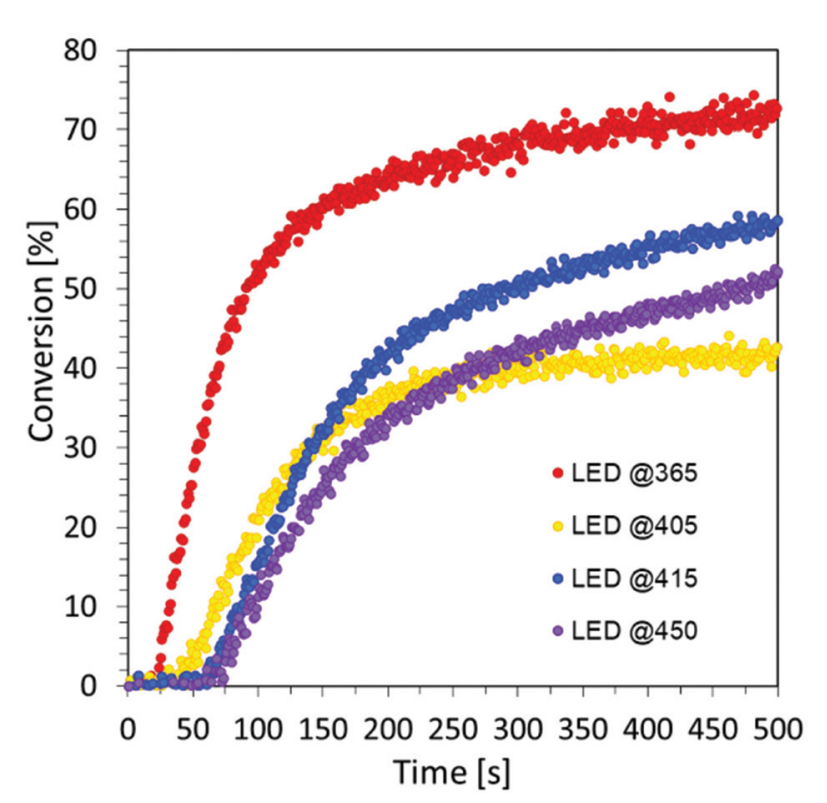

Fig. 11 Free-radical photopolymerization profiles (double bond conversion vs. irradiation time) during the manufacturing of hydrogels, initiated by a two-component photoinitiating system based on IOD (2.0 wt\%) and CA-CDs (0.2 wt\%) under irradiation at $365 \mathrm{~nm}, 405 \mathrm{~nm}$, $415 \mathrm{~nm}$ and $450 \mathrm{~nm}$. Irradiance was measured at a distance of $240 \mathrm{~mm}$ for all sources. 
Table 4 Summary of acrylate monomer conversion rates compared with the basic parameters of the light sources used during the production of hydrogel materials

\begin{tabular}{lllll}
\hline $\begin{array}{l}\text { Conversion } \\
\text { during the } \\
\text { formation } \\
\text { of hydrogels }\end{array}$ & $\begin{array}{l}\mathrm{d} \alpha / \mathrm{d} t \\
{\left[\mathrm{~s}^{-1}\right]}\end{array}$ & $\begin{array}{l}\text { Absorption } \\
\text { at a given } \\
\text { wavelength } \\
\text { [a.u. }\end{array}$ & $\begin{array}{l}\text { Source } \\
\text { of light } \\
\text { [nm] }\end{array}$ & $\begin{array}{l}\text { Intensity } \\
\left(\mathrm{mW} \mathrm{cm}{ }^{-2}\right)\end{array}$ \\
\hline $73 \%$ & 0.84 & 0.265 & @365 & 24.8 \\
$43 \%$ & 0.34 & 0.197 & @ a 405 & 22.0 \\
$59 \%$ & 0.38 & 0.184 & @ a 415 & 24.0 \\
$52 \%$ & 0.30 & 0.143 & @450 & 27.1 \\
\hline
\end{tabular}

Rates of conversion were determined as the maximum slope of the conversion curves obtained by RT-FTIR. Based on the obtained results, we prove the excellent visible light absorption and photosensitizing properties of carbon dots to iodonium salts in light-emitting diodes (LEDs) at visible wavelengths under low light intensity sources during the freeradical photopolymerization processes for the production of hydrogel materials. For this purpose, a composition of HEA acrylate monomer and water in a $1: 1$ mass ratio was prepared, where IOD/CDs (2/0.2 wt\%) were used as the initiating system. Four light sources with different nominal powers were used and the process was conducted in air for $500 \mathrm{~s}$ (Fig. S7 $\dagger$ ), where the composition thickness was $0.5 \mathrm{~mm}$.

The obtained results undoubtedly prove the excellent visible light absorption of C-dots based on the precursor of citric acid, which makes it possible to use them, among others, for the production of hydrogel materials using safe light sources from the visible range. Moreover, the analysis of the data compiled in Table 4 shows that the degree of monomer conversion decreases as the wavelength of light increases.

The main factor affecting the photopolymerization speed and maximal conversion is the light absorption capacity of photo-initiating systems and their value of the molar extinction coefficient at the working light source wavelength. Therefore, the obtained results are consistent with the decrease of the molar extinction coefficient of CDs at longer wavelengths.

In addition, we have shown that we can easily prepare hydrogels via the visible light-photopolymerization of acrylates in water. Moreover, we prove that the synthesized bio-based CDs from citric acid can play a crucial role as photosensitizers under visible light in the above-mentioned experiments. CDs can absorb visible light and transfer energy to the iodonium salt under the working light source and initiate polymerizations at room temperature, under oxygen conditions and even in an aquatic environment. This work provides new perspectives for the generation of radicals in water using bio-based materials and easy-to-set-up reaction conditions.

Proving the catalytic properties of carbon dots. In this work, we present the role of CDs as photocatalysts in photopolymerization reactions. Particularly marked special emphasis is placed on photoredox catalysts leading to highly efficient photoinitiating systems (PISs) for polymerization at low light intensity for use in light-emitting diodes (LEDs) and the possibility of using these PISs based on CDs as a component of photocurable resin for the fabrication of $3 \mathrm{D}$ objects creating the potential to revolutionize computer-guided fabrication of biomaterials as well as design and manufacturing of customized objects tailored to meet the demands of individuals and specific applications. The above studies have proven the possibility of using carbon dots as photosensitizers of iodine salt in hybrid photopolymerization processes by concomitant cationic and free-radical mechanisms. Such an initiating system works according to the photo-oxidation mechanism, where iodine salt as a strong electron acceptor is reduced during the electron transfer process, while carbon dots after light absorption become electron donors in this process. However, in the course of our research, we asked ourselves whether the carbon dots we are considering can also be used in photoinitiating systems, reacting according to the photo-reduction mechanism. In such a system, we need the so-called co-initiator particle (this role is successfully played by tertiary amines), which will be an electron donor for the excited acceptor particle, in our case carbon dots. Popular co-initiators are amines, e.g., methyl diethanolamine which has a low oxidation potential (864 mV (ref. 12)). Consequently, a type II free-radical photoinitiator based on carbon dots and amine (CA-CDs/MDEA) $(0.2 / 2.0 \mathrm{wt} \%)$ was proposed to initiate the free-radical photopolymerization reaction of the HEA monomer in water in a $1: 1$ mass ratio. The study was carried out in air for $300 \mathrm{~s}$, using a LED light source at a wavelength of $405 \mathrm{~nm}$ in the visible range. The graphs showing the dependence of the degree of conversion on time are shown in Fig. 12a, and the values of conversion of acrylate monomers are listed in Table S3.† As can be observed, the polymerization process takes place (blue continuous line), but the monomer conversion values and the kinetics itself are not satisfactory. This has prompted us to further research whether to use carbon dots in a full catalytic cycle, in a three-component system consisting of: iodine salt IOD (2.0 wt\%), amine MDEA (2.0 wt\%) and CA-CDs $(0.2 \mathrm{wt} \%)$. As a reference, the composition of HEA/ water (1:1 wt.) and IOD/MDEA (2.0/2.0 wt\%) was used (Fig. 12a). The values of conversion of acrylate monomers are listed in Table S3. $\dagger$

On comparing the polymerization kinetics of the acrylate monomer during the production of hydrogels for different initiating systems, it is clear that carbon dots are most effective in the full catalytic cycle, where both photo-oxidation and photo-reduction take place and the obtained conversion rate is almost full.

\section{D printing experiments}

3D printing is undoubtedly a relevant topic not only among scientists, but also globally. Application of the 3D printing process is no longer limited to the chemical industry, but increasingly conquers other fields, including modern medicine. ${ }^{77}$ That is why we decided to test various carbon dots for their application in 3D printing as a part of initiation systems. 
(a)

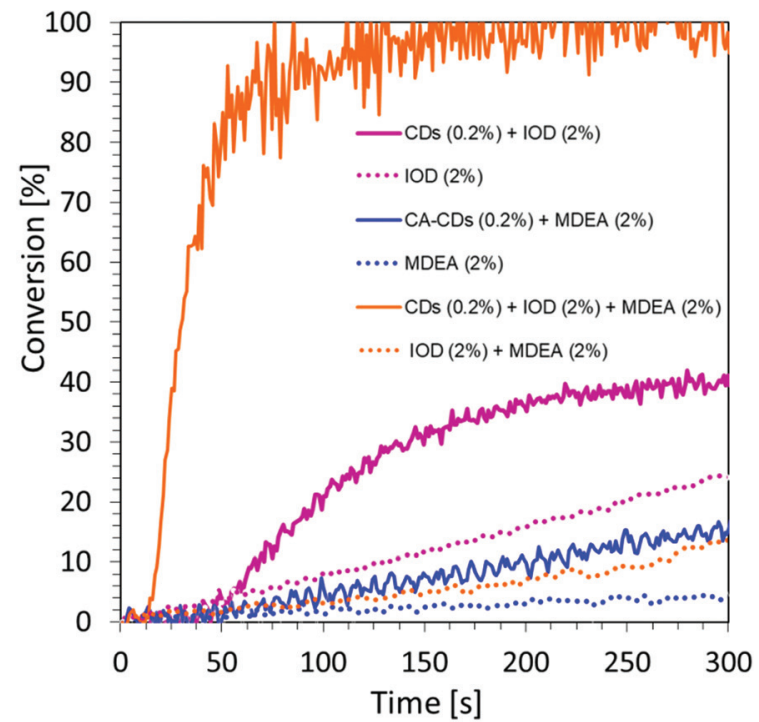

(b)

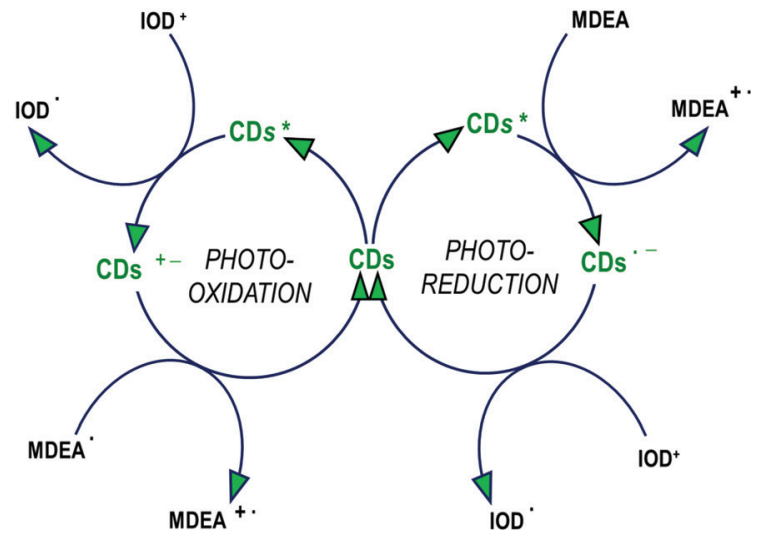

Fig. 12 (a) Free-radical photopolymerization profiles (double bond conversion vs. irradiation time) during the manufacturing of hydrogels, initiated by various photoinitiating systems based on IOD (2.0 wt\%) and CA-CDs (0.2 wt\%) (continuous line), together with appropriate references (dotted line). As a control reference, a mixture of HEA/water (1.1 wt\%) and MDEA amine (2.0 wt\%) was used. Irradiation source: $405 \mathrm{~nm}$ and (b) full catalytic cycle with the use of CDs.

In this study, we have proven for the first time the possibility of using carbon dots as an effective component of the photoinitiating system for 3D printing application, using cationic photopolymerization of vinyl monomers. For this purpose, we prepared a composition consisting of a vinyl monomer TEGDVE and the IOD/N-doped-CA-CD initiating system (2.0/0.2 $\mathrm{wt} \%$ ) and performed 3D printing under a laser source of light @405 nm (spot diameter $50 \mu \mathrm{m}$ ) with an intensity around $90 \mathrm{~mW} \mathrm{~cm}^{-2}$. The photo of the obtained printout is shown in Fig. 13 and Photo S2. $\uparrow$ C-DOT inscription with a significant thickness of $\sim 0.5 \mathrm{~mm}$ and excellent spatial resolution (only limited by the size of the laser diode beam: spot of $50 \mu \mathrm{m}$ ) was achieved.

\section{D printing of hydrogels in aqueous plotting media}

As mentioned above, the use of $3 \mathrm{D}$ printing is currently not only limited to the chemical industry, but is also increasingly

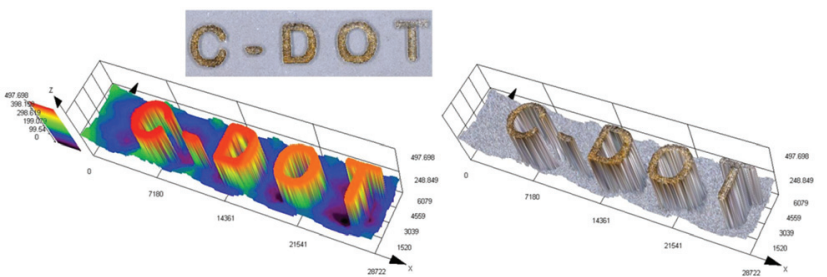

Fig. 13 3D inscription made by the cationic photopolymerization of a TEGDVE monomer in the presence of a two-component photoinitiating system based on $\mathrm{N}$-doped-CA-CDs (0.2 wt\%) and IOD (2 wt\%).

used in biomedicine. Hydrogel materials can be produced through photopolymerization in several ways, e.g. vat polymerization, using fast prototyping techniques, such as photolithography or 3D printing. However, due to its high precision, high efficiency for a single product and convenient operation, 3D printing has recently become the main method of producing hydrogel materials (Photo S1 $\dagger$ ).

The challenge in $3 \mathrm{D}$ printing of hydrogel materials is to choose the suitable photosensitive composition and the type of initiating system, which should be well soluble in an aqueous medium, ensuring the use of visible light sources. Therefore, we created a system of the acrylate monomer HEA and water in a 1:1 mass ratio together with the initiating system IOD/CDs $(2 / 0.2 \mathrm{wt} \%)$ to produce hydrogel materials with a defined structure under a laser source of light @405 nm (spot diameter $50 \mu \mathrm{m}$ ) with an intensity around $90 \mathrm{~mW} \mathrm{~cm}^{-2}$. The photos of the obtained hydrogel printout are shown in Fig. 14. In the results of this experiment, a hydrogel printout of $\sim 2.5 \mathrm{~mm}$ thickness and excellent optical resolution was obtained. Therefore, water soluble nano-photoinitiating systems based on carbon dots hold great promise for environmentally-benign synthesis of useful hydrogel materials under low intensity visible irradiation with light emitting diodes (LEDs). We believe that these results can provide insights into the research done in this field so far, which can pave the way for further progress in this topic of far-fetched social significance. Therefore, carbon dots (C-Dots) are promising new materials for the development of biocompatible photosensitizers for hydrogel production in aqueous solution.

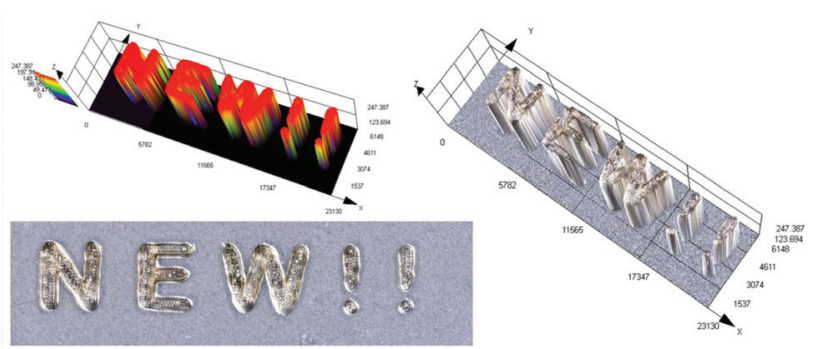

Fig. 14 3D hydrogel inscription made by the free-radical photopolymerization of a HEA/water (1/1 wt\%) mixture in the presence of a two-component photoinitiating system based on CA-CDs (0.2 wt $\%)$ and IOD (2 wt\%). 


\section{Conclusions}

Herein, the first utilization of carbon dots (CDs) as an efficient component of photoinitiating systems (PISs) and photoinitiator catalysts (PICs) for visible-light-induced cationic photopolymerization under a source of low intensity LED irradiation was investigated. A series of structurally different CDs based on citric acid as a precursor (CA-CDs) and containing heteroatom dopants including nitrogen (N-doped-CA-CDs) and sulfur atoms (N,S-co-doped-CA-CDs) were prepared via a 'bottom-up' pyrolysis approach. Moreover, the full path from the synthesis of citric acid-based CDs to their various applications was developed, with special attention paid to the importance of the purification process. Taking advantage of a heteroatom-modulated bottom-up synthetic protocol, CDs were obtained, enabling cationic photopolymerization processes of unsaturated monomers that undergo chain polymerization through the carbon-carbon double bonds, and cyclic monomers that undergo ring-opening polymerization (ROP). This approach links two-component photoinitiating systems (PISs) with further technologies such as coating and 3D printing based on LED sources. Additionally, photopolymerization at different wavelengths covering the electromagnetic spectrum was performed, demonstrating the ability of CDs to act as a component of visible photoinitiating systems (PISs) (Fig. S24†). An interesting aspect of this study is also the description of the $3 \mathrm{D}$ printing experiment of cationic photopolymerization processes initiated by PISs and PICs based on CDs, which offer new opportunities for broadening their applications. Moreover, the development of new and efficient metalfree photoinduced electron transfer (PET) photoinitiator catalyst (PIC) systems regulated by visible light based on CD structural engineering was introduced. Moreover, PICs based on CDs could be used in the production of 3D hydrogel materials using the acrylate monomer HEA and water. Thus, such systems also have great potential in biomedical applications, including the production of hydrogel materials both in situ and via 3D printing, using safe light sources in the visible range. These findings will be assisting researchers in moving forward and exploring the hidden potential of CDs for the development of new photoinitiating systems and in other areas of photochemistry, including interdisciplinary research in 3D printing, which is one of the key pillars of Industry 4.0.

\section{Conflicts of interest}

There are no conflicts to declare.

\section{Acknowledgements}

This research was funded by the Foundation for Polish Science (Warsaw, Poland) TEAM TECH project Grant No. TEAM TECH/ 2016-2/15 (POIR.04.04.00-00-204B/16-00). Additionally, the research of synthesis of CDs was supported by the Polish
National Agency for Academic Exchange (The Bekker Programme No. PPN/BEK/2018/1/00460/U/00001). The authors are also grateful to Mrs Marta Gejewska from the Academic Centre for Materials and Nanotechnology (ACMiN), AGH University of Science and Technology for the TEM analysis used during this study.

\section{References}

1 P. Glöckner, S. Struck, T. Jung and K. Studer, Radiation Curing: Coatings and Printing Inks, Technical Basics, Applications and Trouble Shooting, 2008, vol. 3.

2 P. Fiedor and J. Ortyl, Materials, 2020, 13, 2951.

3 J. Olmsted, J. Phys. Chem., 1979, 83, 2581-2584.

4 A. Bagheri and J. Jin, ACS Appl. Polym. Mater., 2019, 1, 593611.

5 M. Layani, X. Wang and S. Magdassi, Adv. Mater., 2018, 30, e1706344.

6 A. Bagheri, K. E. Engel, C. W. A. Bainbridge, J. Xu, C. Boyer and J. Jin, Polym. Chem., 2020, 11, 641-647.

7 J. Zhang and P. Xiao, Polym. Chem., 2018, 9, 1530-1540.

8 E. Hola, J. Ortyl, M. Jankowska, M. Pilch, M. Galek, F. Morlet-Savary, B. Graff, C. Dietlin and J. Lalevée, Polym. Chem., 2020, 11, 922-935.

9 E. Hola, M. Topa, A. Chachaj-Brekiesz, M. Pilch, P. Fiedor, M. Galek and J. Ortyl, RSC Adv., 2020, 10, 7509-7522.

10 S. C. Ligon, R. Liska, J. Stampfl, M. Gurr and R. Mülhaupt, Chem. Rev., 2017, 117, 10212-10290.

11 Z. Wang, W. Huang, P. Peng and D. E. Fennell, Chemosphere, 2010, 78, 147-151.

12 W. Tomal, M. Pilch, A. Chachaj-Brekiesz, M. Galek, F. Morlet-Savary, B. Graff, C. Dietlin, J. Lalevée and J. Ortyl, Polym. Chem., 2020, 11, 4604-4621.

13 W. Tomal and J. Ortyl, Polymers, 2020, 12, 1073.

14 J. W. Stansbury, J. Esthet. Restor. Dent., 2000, 12, 300-308.

15 M. E. Khosroshahi, M. Atai and M. S. Nourbakhsh, Lasers Med. Sci., 2008, 23, 399-406.

16 N. A. Peppas, J. Z. Hilt, A. Khademhosseini and R. Langer, Adv. Mater., 2006, 18, 1345-1360.

17 J. L. Ifkovits and J. A. Burdick, Tissue Eng., 2007, 13, 23692385.

18 R. Censi, W. Schuurman, J. Malda, G. di Dato, P. E. Burgisser, W. J. A. Dhert, C. F. van Nostrum, P. di Martino, T. Vermonden and W. E. Hennink, Adv. Funct. Mater., 2011, 21, 1833-1842.

19 G. J. Kavarnos and N. J. Turro, Chem. Rev., 1986, 86, 401-449.

20 W. Tomal, M. Pilch, A. Chachaj-Brekiesz and J. Ortyl, Catalysts, 2019, 9, 827.

21 W. Tomal, A. Chachaj-Brekiesz, R. Popielarz and J. Ortyl, RSC Adv., 2020, 10, 32162-32182.

22 E. Hola, M. Pilch, M. Galek and J. Ortyl, Polym. Chem., 2020, 11, 480-495.

23 E. Hola, M. Pilch and J. Ortyl, Catalysts, 2020, 10, 1-28.

24 P. Fiedor, M. Pilch, P. Szymaszek, A. Chachaj-Brekiesz, M. Galek and J. Ortyl, Catalysts, 2020, 10, 284. 
25 J. Ortyl, P. Fiedor, A. Chachaj-Brekiesz, M. Pilch, E. Hola and M. Galek, Sensors, 2019, 19, 1668.

26 M. Topa, F. Petko, M. Galek and J. Ortyl, Sensors, 2020, 20(11), 3043.

27 J. Zhang, N. Zivic, F. Dumur, P. Xiao, B. Graff, J. P. Fouassier, D. Gigmes and J. Lalevée, Polym. Chem., 2018, 9, 994-1003.

28 M. L. Liu, B. B. Chen, C. M. Li and C. Z. Huang, Green Chem., 2019, 21, 449-471.

29 C. Xia, S. Zhu, T. Feng, M. Yang and B. Yang, Adv. Sci., 2019, 6, 1901316.

30 M. Liu, Nanoarchitectonics, 2020, 1, 1-12.

31 J. Wang, P. Zhang, C. Huang, G. Liu, K. C. F. Leung and Y. X. J. Wáng, Langmuir, 2015, 31, 8063-8073.

32 Y. Song, X. Yan, Z. Li, L. Qu, C. Zhu, R. Ye, S. Li, D. Du and Y. Lin, J. Mater. Chem. B, 2018, 6, 3181-3187.

33 F. Yan, Y. Jiang, X. Sun, J. Wei, L. Chen and Y. Zhang, Nano Res., 2020, 13, 52-60.

34 Y. Wang, Y. Zhu, S. Yu and C. Jiang, $R S C A d v ., 2017,7$, 40973-40989.

35 V. Strauss, J. T. Margraf, C. Dolle, B. Butz, T. J. Nacken, J. Walter, W. Bauer, W. Peukert, E. Spiecker, T. Clark and D. M. Guldi, J. Am. Chem. Soc., 2014, 136, 17308-17316.

36 J. Zhang and S. H. Yu, Mater. Today, 2016, 19, 382-393.

37 L. Li and T. Dong, J. Mater. Chem. C, 2018, 6, 7944-7970.

38 V. Sharma, P. Tiwari and S. M. Mobin, J. Mater. Chem. B, 2017, 5, 8904-8924.

39 H. Tao, K. Yang, Z. Ma, J. Wan, Y. Zhang, Z. Kang and Z. Liu, Small, 2012, 8, 281-290.

40 F. Chen, W. Gao, X. Qiu, H. Zhang, L. Liu, P. Liao, W. Fu and Y. Luo, Front. Lab. Med., 2017, 1, 192-199.

41 W. Kasprzyk, S. Bednarz and D. Bogdał, Chem. Commun., 2013, 49, 6445-6447.

42 W. Kasprzyk, S. Bednarz, P. Zmudzki, M. Galica and D. Bogdał, RSC Adv., 2015, 5, 34795-34799.

43 W. Kasprzyk, T. Świergosz, S. Bednarz, K. Walas, N. V. Bashmakova and D. Bogdał, Nanoscale, 2018, 10, 13889-13894.

44 V. Strauss, H. Wang, S. Delacroix, M. Ledendecker and P. Wessig, Chem. Sci., 2020, 11, 8256-8266.

45 C. I. M. Santos, I. F. A. Mariz, S. N. Pinto, G. Gonçalves, I. Bdikin, P. A. A. P. Marques, M. G. P. M. S. Neves, J. M. G. Martinho and E. M. S. Maçôas, Nanoscale, 2018, 10, 12505-12514.

46 X. Jia and X. Ji, RSC Adv., 2015, 5, 107270-107275.

47 J. Jiang, G. Ye, Z. Wang, Y. Lu, J. Chen and K. Matyjaszewski, Angew. Chem., Int. Ed., 2018, 57, 1203712042.

48 C. Kütahya, P. Wang, S. Li, S. Liu, J. Li, Z. Chen and B. Strehmel, Angew. Chem., 2020, 59, 3166-3171.

49 Q. Wang, L. Hu, Z. Cui, P. Fu, M. Liu, X. Qiao and X. Pang, ACS Appl. Mater. Interfaces, 2020, 12, 42161-42168.

50 S. Kalytchuk, L. Zdražil, M. Scheibe and R. Zbořil, Nanoscale, 2020, 12, 8379-8384.

51 K. Bazaka, M. V. Jacob and K. Ostrikov, Chem. Rev., 2016, 116, 163-214.
52 T. D. Ngo, A. Kashani, G. Imbalzano, K. T. Q. Nguyen and D. Hui, Composites, Part B, 2018, 143, 172-196.

53 H. Bennett and G. Oliver, J. Chem. Educ., 1993, 70, A25.

54 P. G. Rouxhet and M. J. Genet, Surf. Interface Anal., 2011, 43, 1453-1470.

55 M. Fantauzzi, B. Elsener, D. Atzei, A. Rigoldi and A. Rossi, RSC Adv., 2015, 5, 75953-75963.

56 A. D. Wagner, A. V. Naumkin, A. Kraut-Vass, J. W. Allison, C. J. Powell and J. R. J. Rumble, NIST Standard Reference Database 20, http:/srdata.nist.gov/xps/.

57 C. Carbonaro, R. Corpino, M. Salis, F. Mocci, S. Thakkar, C. Olla and P. Ricci, C, 2019, 5, 60.

58 J. Tang, J. Zhang, Y. Zhang, Y. Xiao, Y. Shi, Y. Chen, L. Ding and W. Xu, Nanoscale Res. Lett., 2019, 14, 1-10.

59 G. E. Lecroy, F. Messina, A. Sciortino, C. E. Bunker, P. Wang, K. A. S. Fernando and Y. P. Sun, J. Phys. Chem. C, 2017, 121, 28180-28186.

60 M. A. Tasdelen, J. Lalevée and Y. Yagci, Polym. Chem., 2020, 11, 1111-1121.

61 J. Lalevée, M. A. Tehfe, A. Zein-Fakih, B. Ball, S. Telitel, F. Morlet-Savary, B. Graff and J. P. Fouassier, ACS Macro Lett., 2012, 1, 802-806.

62 A. Balcerak, D. Kwiatkowska, K. Iwińska and J. Kabatc, Polym. Chem., 2020, 11, 5500-5511.

63 M. A. Tehfe, J. Lalevée, X. Alionas and J. P. Fouassier, Macromolecules, 2009, 42, 8669-8674.

64 P. P. Romańczyk and S. S. Kurek, Electrochim. Acta, 2017, 255, 482-485.

65 B. Strehmel, Z. Phys. Chem., 2014, 228, 129-153.

66 E. Andrzejewska, D. Zych-Tomkowiak, M. Andrzejewski, G. L. Hug and B. Marciniak, Macromolecules, 2006, 39, 3777-3785.

67 K. Haraguchi, J. Stem Cells Regener. Med., 2012, 8, P2P11.

68 M. Chen, M. Zhong and J. A. Johnson, Chem. Rev., 2016, 116, 10167-10211.

69 Q. Cao, T. Heil, B. Kumru, M. Antonietti and B. V. K. J. Schmidt, Polym. Chem., 2019, 10, 5315-5323.

70 S. E. Shim, Y. Shin, J. W. Jun, K. Lee, H. Jung and S. Choe, Macromolecules, 2003, 36, 7994-8000.

71 F. Le Quéméner, D. Subervie, F. Morlet-Savary, J. Lalevée, M. Lansalot, E. Bourgeat-Lami and E. Lacôte, Angew. Chem., 2018, 130, 969-973.

72 X. Huang, X. Wang and Y. Zhao, Dyes Pigm., 2017, 141, 413-419.

73 A. S. Hoffman, Adv. Drug Delivery Rev., 2012, 64, 18-23.

74 R. Sanna, V. Alzari, D. Nuvoli, S. Scognamillo, S. Marceddu and A. Mariani, J. Polym. Sci., Part A: Polym. Chem., 2012, 50, 1515-1520.

75 F. Ahmadi, Z. Oveisi, M. Samani and Z. Amoozgar, Res. Pharm. Sci., 2015, 10, 1-16.

76 C. Arakawa, R. Ng, S. Tan, S. Kim, B. Wu and M. Lee, J. Tissue Eng. Regener. Med., 2017, 11, 164-174.

77 A. Ghilan, A. P. Chiriac, L. E. Nita, A. G. Rusu, I. Neamtu and V. M. Chiriac, J. Polym. Environ., 2020, 28, 13451367. 